\title{
Wireless Systems and Printed Antennas
}

\author{
Rod Waterhouse and Dalma Novak
}

Pharad, Maryland, USA

\subsection{INTRODUCTION}

Wireless systems are playing an ever increasingly important role in society. Whether they are used to assist in the distribution/collection of large amounts of information or to make home entertainment systems more convenient, wireless systems are becoming more and more integrated into daily activities. The utilization of wireless technology is not currently confined to either the commercial or the military sectors. In fact, it seems that both sectors are striving for wireless solutions to enhance their resources, or simply to make their product more readily accessible and useful to the consumer.

One of the important enabling technologies for wireless communications is the transducer, which converts guided energy to radiated energy (and vise versa): the antenna. Although advances in antenna engineering cannot be credited for the globalization of wireless technologies, it still plays an important role, whether it provides an aesthetically pleasing solution or helps improve the overall radio frequency (RF) link budget, or allows multiple users to utilize the single interface and thereby increase the capacity. It is undeniable that good radiator engineering allows for better wireless systems.

Figure 1.1 shows a 'snapshot' of present and future commercial wireless systems, highlighting cellular and satellite communications, as well as the extremely popular wireless local area network (WLAN) and short-range connectivity systems such as Bluetooth. Also shown is the quandary facing next-generation systems and how they were interface with a variety of technologies including computers and small handsets.

Figure 1.2 shows the frequency spectrum indicating current and next-generation wireless systems between $300 \mathrm{MHz}$ and $100 \mathrm{GHz}$. As can be seen, there are a plethora of wireless systems utilizing various advantages of the different spectra. For example, systems at the 


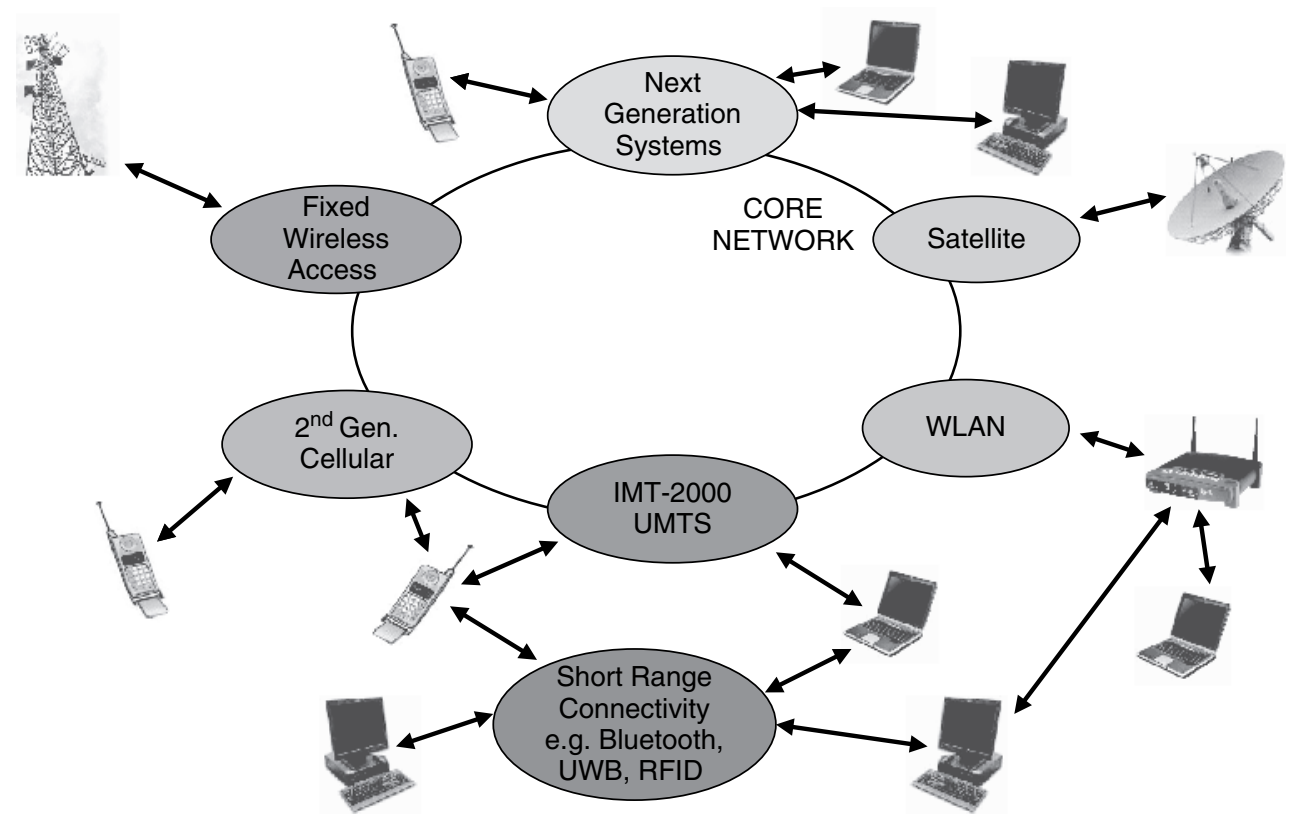

Figure 1.1 Schematic showing a variety of present-day and future wireless systems.

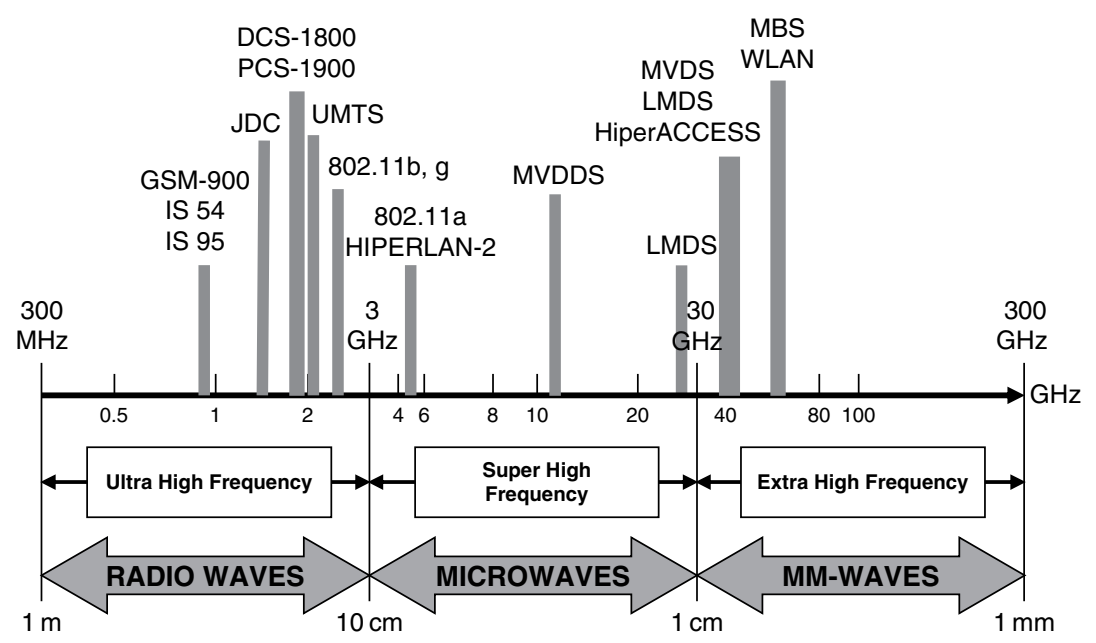

Figure 1.2 The frequency spectrum showing many wireless systems.

lower end of the frequency spectrum have better propagation and mobility characteristics, whereas the higher frequency applications can transmit significantly more information. As can be seen from Figures 1.1 and 1.2, there are many issues with future wireless systems, not just related to antenna engineering. Not only do these wireless networks have to be seamlessly interconnected to wired networks, but perhaps with different radio networks and interfaces. Ideally the next-generation radio network interface will have a degree of flexible control that can adapt to different modulation formats and air interface access formats. 
This chapter serves as an introduction to the remainder of the book. In particular the authors will focus on aspects and issues that the antenna engineer needs to be aware of to ensure the ultimate solution is obtained for the problem at hand. In Section 1.2 some present-day examples of wireless systems will be presented. They will focus on some less conventional wireless applications to give the reader an appreciation that the commercial and military sectors are not just interested in cellular systems. From this investigation, it will be seen that there are many and sometimes conflicting requirements for the radiator, and as the antenna is typically the final component to be designed in the RF link, the antenna engineer is usually burdened with an extremely difficult task that may involve attempting to break the laws of physics. In Section 1.3 some new platforms are presented that require efficient wireless solutions: unmanned vehicles and body wearable solutions. Both platforms present very challenging issues to the antenna designer. Based on the reviewed systems and platforms, in Section 1.4 the authors will focus on some general requirements for antennas. Section 1.5 will give a brief review of printed antennas and summarize the characteristics of the different classes. Finally in Section 1.6 the intention of the rest of the book will be summarized, showing how the technologies presented can help the antenna engineer deliver the best radiating solution for a particular wireless system.

\subsection{EXAMPLES OF WIRELESS SYSTEMS}

Conventional wireless communications systems (cellular/mobile) continue to be the cornerstone of society, with more bandwidth hungry applications being offered to the public (for example the streaming video), and presently these applications are readily being accepted by the public as 'must have' technologies. Satellite systems, for communications, broadcasting, and positioning (global positioning satellite (GPS) system), seem to be experiencing a bit of a renaissance, with recent applications adding to the overall importance of wireless technologies. The WLAN is another example of wireless technology that is having a dramatic impact on society, enabling low-cost wireless solutions that are easy to install in coffee shops, homes, and communal areas for consumers. The unlicensed IEEE 802.11.x systems have opened the door to low-cost wireless systems that are inevitably challenging 'conventional' systems. Excellent reviews of these wireless systems can be found in References [1] to [4]. There are other wireless communication systems that are receiving a lot of attention and here a summary of a few of these is provided. After reading these systems/applications, it will hopefully be apparent to the reader that there are many diverse applications requiring all forms of radio technology, some applicable to both military and commercial interests.

\subsubsection{RFID/Asset Tracking}

The location and tracking of inventory and assets is a critical activity for many businesses. Passive and active RFID (radio frequency identification) is a well-established technology that provides a means of contactless identification and is now used extensively in a range of industries. Organizations are quickly recognizing the benefits of RFID as a solution for inventory control (identification and tracking) that can bring a rapid return on investment (ROI). In addition, RFID tags are already being used for applications as diverse as: theft prevention; the identification of animals and humans; automating production systems; electronic toll 
collection and traffic automation; intermodal container identification and tracking; control of access to vehicles, buildings, and parking areas; car immobilizing (security); and automatic automobile fuel dispensing.

Rapidly expanding markets for RFID technology such as supply chain management have already emerged, with the US market for such technology estimated at \$1.1 billion in 2001 . This is expected to increase dramatically over the next few years, with the market expected to more than double to $\$ 2.3$ billion by 2005 . Major commercial companies such as WalMart and Tesco have recently announced their intention to deploy RFID tag systems extensively throughout their organizations. In addition, recent US Department of Defense (DoD) policy has indicated implementation requirements for RFID technology which commenced in early $2005[5,6]$.

RFID is an electronic labelling system that uses radio frequencies to identify goods quickly and precisely. Information from the tag attached to an item or set of goods is obtained from a reader or interrogator via a radio link. The system provides much greater flexibility over barcode inventory control systems since there are no line-of-sight communication requirements and physical contact between the tag and reader is not required. The tags themselves differ substantially in terms of size, memory capacity, physical construction, and distance over which they can be operated. Consequently tags are often custom designed to meet the needs of specific applications and are therefore typically proprietary in nature. Readers manage the data communications process with the tags, which often utilize a proprietary protocol based on the unique characteristics of the tag technology employed.

While the commercial sector has been quick to adopt RFID for inventory control, the widespread usage of such technology in the military environment has been hampered by the lack of a common set of RFID standards and communication protocols. Numerous RFID vendors exist who typically use their own proprietary tag and reader technologies including communication protocols. This means that tags from one manufacturer require readers from the same vendor and readers from one manufacturer cannot read tags from other vendors. In addition, while national regulatory bodies allocate different radio transmission frequencies for RFID, there is no one global standard. Compatibility with local, national, and international regulations is a key issue in the management of inventory across borders.

RFID technology uses frequencies within the range of $50 \mathrm{kHz}$ to $5.9 \mathrm{GHz}$ where the type of application determines the frequency that is used for a specific system solution, and, in turn, finds which reader and tag will provide the best solution in terms of range, reliability, data transfer rate, and cost. Asset tracking applications that require read ranges greater than 10 feet are based on either backscatter techniques (where a small portion of the reader's RF energy is reflected from the tag, encoded with information stored in it) or two-way active tags (for higher value asset control) and operate at frequencies in the $433 \mathrm{MHz}, 915 \mathrm{MHz}$, $2.45 \mathrm{GHz}$, and $5.8 \mathrm{GHz}$ bands.

The form factor and frequency of the RFID tag vary widely depending on the application, and environmental factors also play a significant role. For example, a tag operating at 13.56 $\mathrm{MHz}$ has a size of $7.6 \mathrm{~cm} \times 4.5 \mathrm{~cm}$ while one operating at $2.45 \mathrm{GHz}$ has a size of only $6 \mathrm{~cm} \times 1 \mathrm{~cm}$. Figure 1.3 shows a commercially available active RFID tag developed for conventional corrugated shipping cartons [7]. As shown in this photograph, the integration of RFID transponders in self-adhesive functional components expands their potential uses. When combined with labels, RFID transponders can be fastened to virtually any substrate with either permanent or removable adhesion, visibly or invisibly. For extreme application requirements, RFID labels are coated with highly durable polyurethane resin. Figure 1.3 also shows that the RFID antenna and electronics are bonded on to a flexible acrylic substrate. 


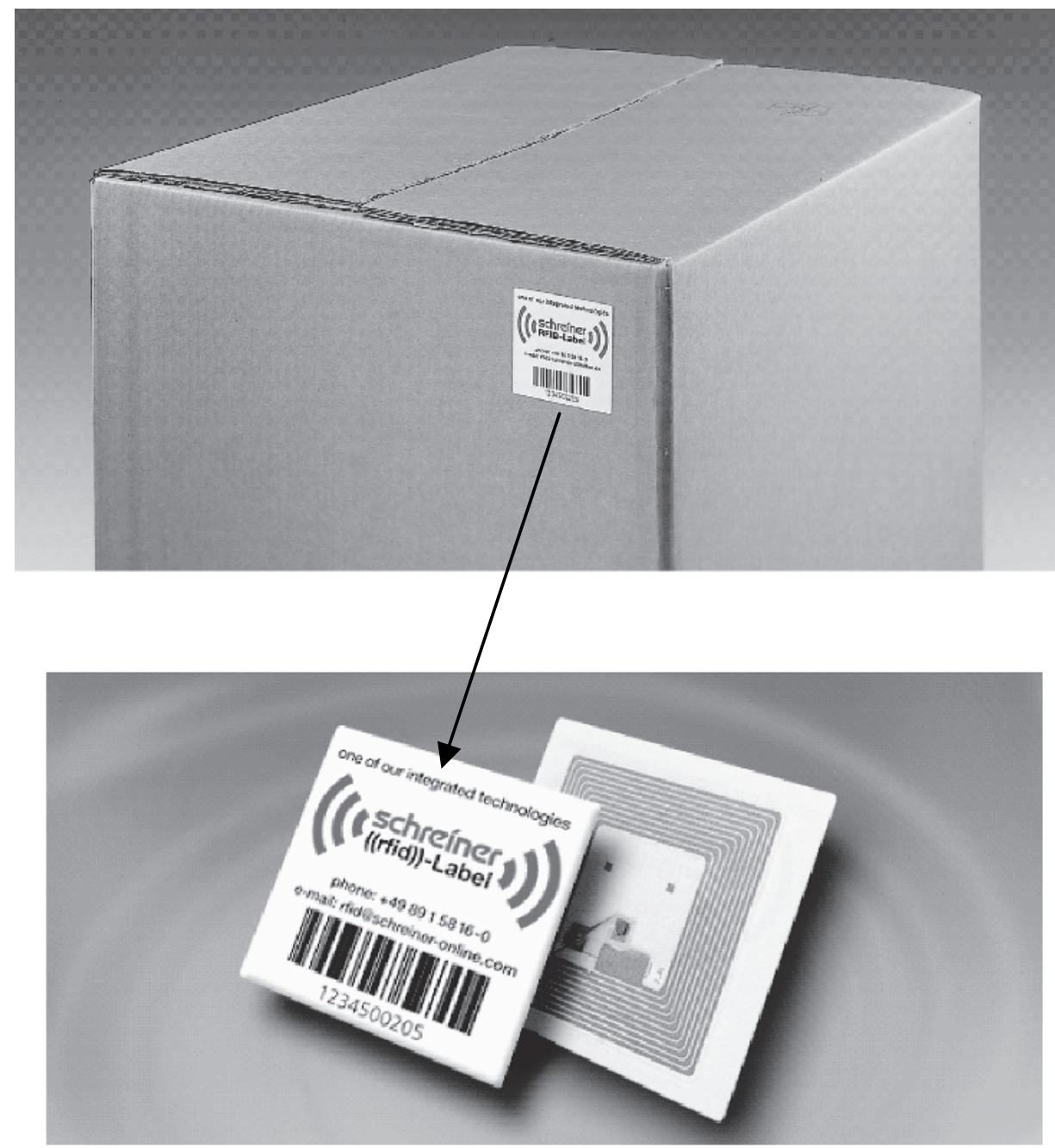

Figure 1.3 Photograph of a commercially available RFID label for shipping cartons [7].

Since RFID systems do not require direct line-of-sight for communications, the tag can also be concealed and embedded directly in the casing of the product.

Figure 1.4 shows a schematic of an RFID base station module for $2.4 \mathrm{GHz}$ utilizing a dual polarized patch antenna for improved performance. The radiator is easily integrated with the required RF and low-frequency electronics and is protected from the environment by a radome.

\subsubsection{Automatic Identification System (AIS)}

In the last five years, an Automatic Identification System (AIS) shipboard broadcast transponder system has gone from development to deployment in large ocean-going vessels. The AIS is a communication protocol developed, and being extended (for Class B), by the 


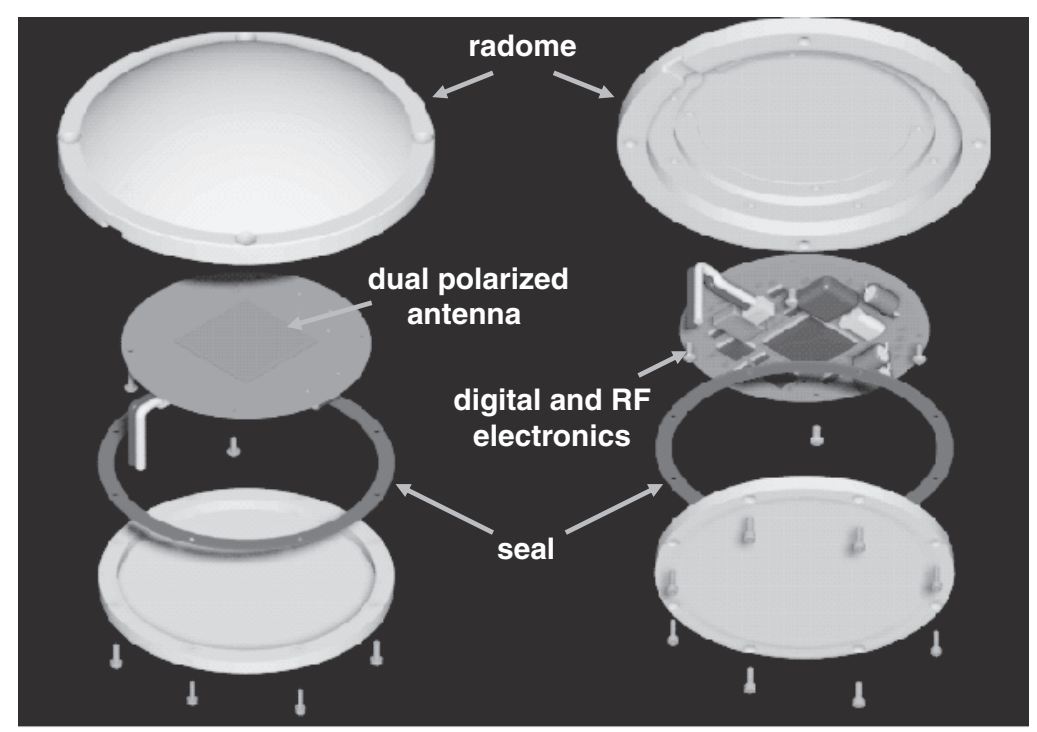

Figure 1.4 Schematic of an RFID base station using patch technology.

International Maritime Organization (IMO), International Telecommunication Union (ITU), and International Electrotechnical Commission (IEC). A vessel outfitted with AIS equipment continually transmits its identification, position, course, speed, and other data to all other nearby ships in the very high frequency (VHF) maritime band. The AIS is meant to accomplish the following:

- It operates as a collision avoidance system as a ship-to-ship navigation aid.

- It aids search and rescue operations when supporting aircraft are outfitted with AIS receivers.

- It allows coastal states to obtain information about incoming vessels and their cargo.

- It allows ports to better manage their waters' traffic when integrated into a Vessel Tracking System (VTS).

The large-scale use of AIS equipment is a key foundation for improving waterways management and building a waterway and coastline border security programme. Requiring all blue water vessels to deploy a common AIS sensor infrastructure will allow countries to maintain persistent surveillance of large and small vessel movements. Integrated with a VTS, this border security system will have a priori data available on the identity of vessels entering certain waters, knowledge of movement within the sensor system, knowledge of previous ports visited, and knowledge of potential at-sea rendezvous of multiple vessels.

In accordance with the International Convention for the Safety of Life at Sea (SOLAS) as amended in 2000, AIS transponders were mainly deployed in vessels larger than 300 tons. Pursuant to new rules published in 2003 by the United States Coast Guard, the Department of Homeland Security's lead agency for maritime homeland security, deployment of AIS was required for considerably smaller commercial vessels, as small as 65 feet or 100 tons, by the end of 2004. Clearly, larger vessels pose the greatest consequence resulting from 
a collision. However, these larger vessels represent but a fraction of vessels on the water. The preponderance of maritime traffic is smaller commercial vessels and recreational craft, which are not currently required to deploy AIS equipment. The high cost of currently available AIS equipment is an impediment to the broad adoption of AIS equipment for these smaller maritime vessels. In fact, when the US Coast Guard sought comments regarding the expansion of AIS to vessels not previously required, it amended the interim rules such that only a smaller set of SOLAS certified vessels are required to carry an AIS. However, the US Coast Guard still envisions a time when all vessels operating in US waters carry AIS and is seeking a lower cost AIS solution.

A block diagram of the electrical/physical architecture is shown in Figure 1.5. The position data originates from an integrated GPS receiver which obtains position and time data that is then processed to calculate course data. The GPS receiver is connected to an external GPS antenna via a separately supplied cable. The GPS data are transferred to a central processing unit (CPU), likely a microcontroller, where the information is used to construct AIS transmissions and is used as a variable in the collision avoidance algorithms. External AIS and DSC (digital selective calling) transmissions are received via three independent receivers within the AIS Class B Basic device. A single VHF transmitter is used for all transmissions from this device. The transceiver is connected to an external VHF antenna via a separately supplied cable. The AIS/DSC messages are deconstructed and formatted in a programmable logic device. Power is supplied to all the electronics via a supply that converts the vessel's $12 \mathrm{~V}$ DC power to the appropriate voltages for the electronic circuits.

A central processing function is provided by a microcontroller. This microcontroller executes collision avoidance algorithms using the position and track data from the host vessel and the AIS information reported by other vessels. The microcontroller manages the user interface, including reading the user input via buttons and driving the display information. The microcontroller works with the programmable logic device to aid in the construction and formatting of AIS transmissions/reception of AIS reports from other vessels.

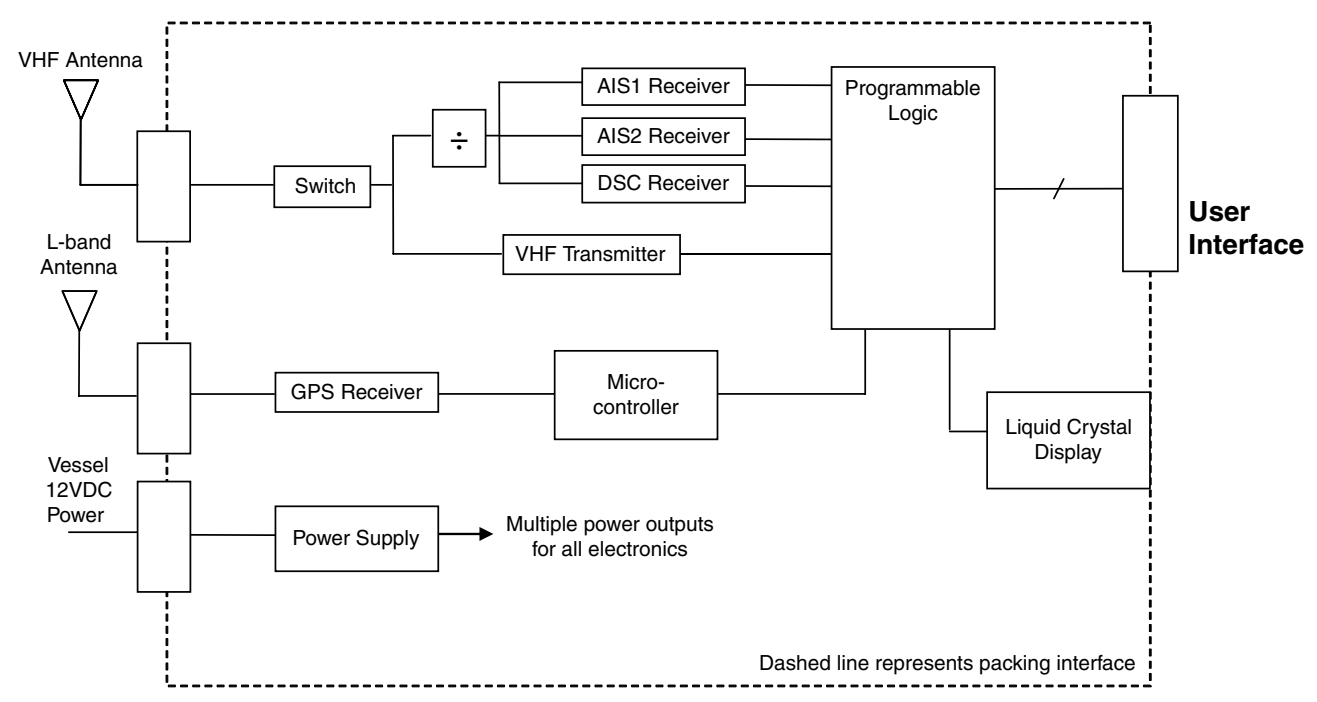

Figure 1.5 Block diagram of the AIS device functional and physical architecture. 


\subsubsection{Joint Tactical Radio System (JTRS)}

One of the prime objectives of the Joint Tactical Radio System (JTRS) is to realize a single software radio capable of processing different waveforms from many different RF services over a wide band of frequencies [8]. The JTRS waveforms supported in the 2 $\mathrm{MHz}-2 \mathrm{GHz}$ frequency range include future Wideband Networking Waveform (WNW), Single Channel Ground Air Radio System (SINCGARS), Ultra High Frequency (UHF) SATCOM, High Frequency (HF) Independent Side Band (ISB) with Automatic Link Establishment (ALE), Link-16, Identification Friend or Foe (IFF), Digital Wideband Transmission System (DWTS), Soldier Radio, Wireless Local Area Network (WLAN), Cellular Radio, Personal Communication Services (PCS), and future expansion of Mobile Satellite Services (MSS) [9]. The implementation of these services in JTRS will require the development of a wideband, airborne antenna platform including a very sophisticated filtering procedure. The radiating structure is the key enabling technology for the proposed software defined radio since wideband amplifier technologies as well as the supporting control software for these communication links are reasonably well advanced. The schematic in Figure 1.6 highlights the multitude of communication levels and therefore the overall complexity of communications in a battlefield environment.

\subsubsection{Mobile ad hoc Network (MANET)}

While ad hoc networks are of great interest for military and tactical applications, other applications have also emerged. These include commercial applications such as an infrastructureless network of notebook computers in temporary offices or a conference/campus

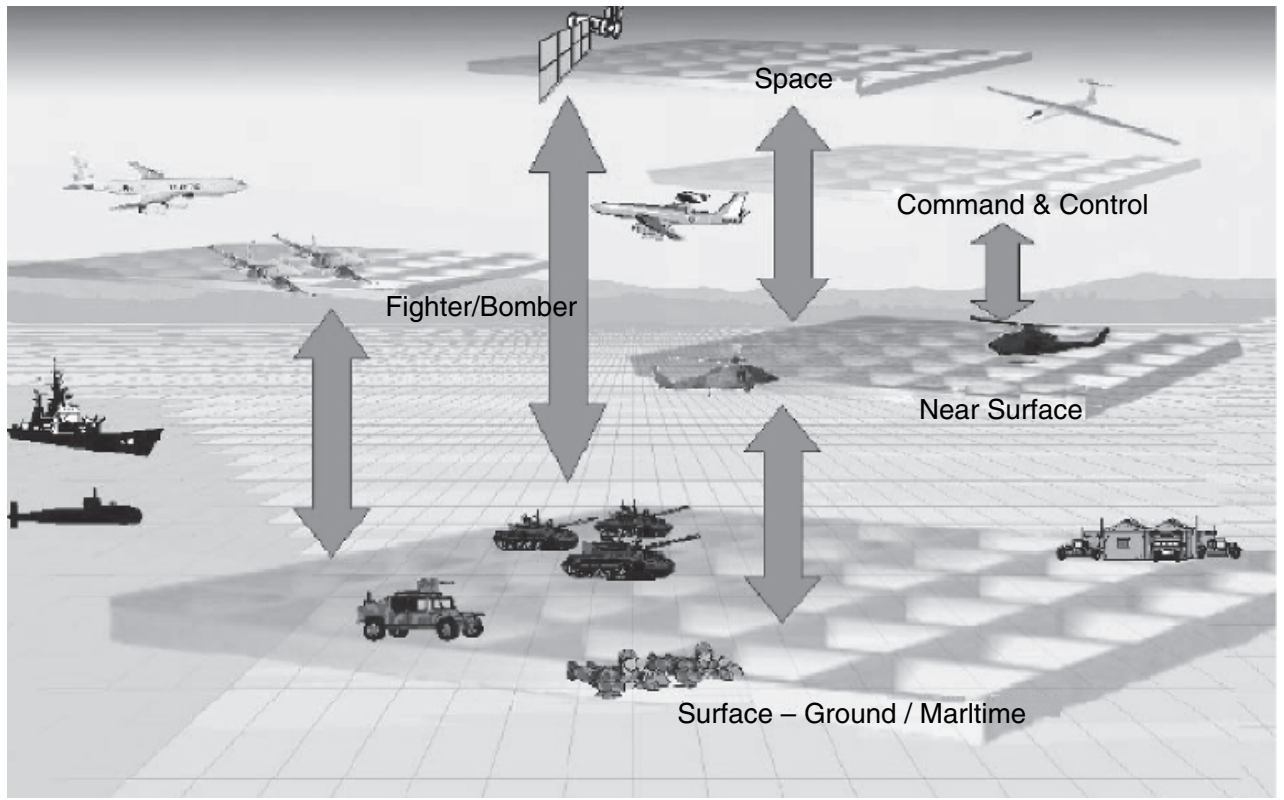

Figure 1.6 Schematic showing the levels of communications in a battlefield environment. 
setting, as well as situations where service is to be provided without the visible presence of a wired backbone. There is also a growing interest in large-scale sensor network applications where hundreds or thousands of sensors may be scattered throughout a city for biological detection. Thus, this technology has great potential where it can reduce the cost and time associated with installing a fixed infrastructure.

In the commercial market, the proliferation of wireless local area networks, and wireless personal area networks, such as Bluetooth, pave the way for ad hoc networking as a key enabling technology. Ad hoc networking will have the ability to open up a range of business, gaming, and content-distribution applications that will lead to increased network usage and potential revenue. In addition, a number of companies are already including ad hoc networking concepts in their products based on wireless mesh networks, including MeshNetworks, Flarion, and Cybiko. Ad hoc networks are also being considered for the extension of the cellular airinterface, leading to improvements in cellular capacity and coverage. As a result, ad hoc network technologies have the opportunity to tap into a multibillion industry in the evolution towards next-generation cellular communication systems. A schematic highlighting a typical ad hoc wireless system architecture is shown in Figure 1.7.

A mobile ad hoc network (MANET) is an autonomous system of mobile hosts (also serving as routers) connected by wireless links that form a communication network with no wired backbone infrastructure [10]. This is in contrast to the conventional single-hop cellular network model where fixed prelocated cell sites and base stations (BSs) are installed as access points and communication between two mobile nodes relies completely on the wired backbone and BSs. Since the geographic location of all network nodes can change in a MANET, the network topology as well as the paths between two terminating points are highly dynamic. In addition, there is no predetermined organization of available links between users; thus an ad hoc network forms automatically and adapts to the changing network, with individual nodes responsible for dynamically discovering other nodes that they can directly communicate with. Since not all nodes can directly communicate with each other, they are also required to relay packets on behalf of other nodes to deliver data across the network. A MANET is thus an example of a multihop wireless network.

Ad hoc networks are of particular use where infrastructure is either not available or cannot be relied on in times of emergency. A MANET builds on the concept of packet switched radio networks that date back to the early 1970s with the US Department of Defense (DoD) funded programs to investigate such concepts for the military and enable mobile communication in infrastructureless, hostile battlefield environments where the nodes forming the network could be soldiers, tanks, or aircraft. While MANETs are of still great

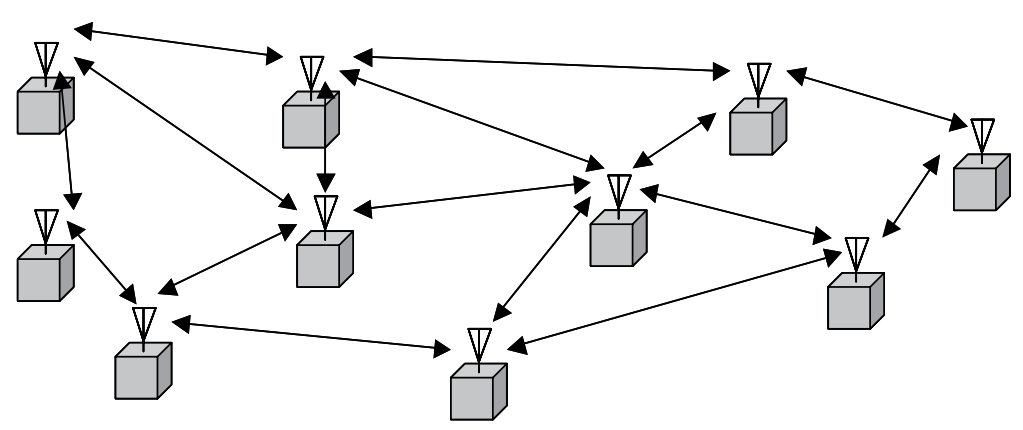

Figure 1.7 A typical architecture for a mobile wireless ad hoc network. 
interest for military and tactical applications (e.g. law enforcement, emergency rescue, or exploration missions), other applications have since emerged. These include commercial applications such as an infrastructureless network of notebook computers in temporary offices or a conference/campus setting, as well as situations where service is to be provided without the visible presence of a wired backbone. There is also a growing interest in large-scale sensor network applications where hundreds or thousands of sensors may be scattered throughout a city for biological detection.

Multicasting in MANETs, where messages are sent from one node to multiple recipients, can efficiently support a variety of applications and is typically the most characteristic operation of MANETs used in military and tactical applications. In these scenarios, multicasting is generally used for disseminating important and confidential information. Therefore multicasting routing protocols need to ensure a reliable message delivery, an area that is actively being pursued [11]. Since the routes between nodes in a MANET are typically multihop in nature, a global routing approach that operates across the whole network and maintains and updates the routing structure whenever changes occur in the system is required. Due to the dynamic nature of the mobile ad hoc environment, the routing protocol must be able to adapt dynamically to changes in the network topology as well as optimize the path between any two nodes. A key challenge in ad hoc network routing is achieving such a goal while also being constrained by the nature of the network itself, since nodes in MANETs typically have limited lifetimes and processing capabilities and the wireless medium also has limited bandwidth.

Due to their inherent characteristics there are many challenges in implementing mobile wireless ad hoc networks that are currently being pursued across all layers of the network. The physical layer has to be able to adapt to rapid changes in the characteristics of the link between two nodes. The medium access control (MAC) layer needs to minimize collisions, allow fair access, and transport data reliably over the shared wireless links. The network layer must determine and distribute information used to calculate routing paths in a way that maintains efficiency when links change often and bandwidth is at a premium. Thus ad hoc networking is a cross-layer design challenge. An emerging challenge is the design of MANETs that can exploit the properties of new hardware technologies. One example is that of smart or beamforming antennas, which do not have a fixed antenna pattern but can adapt to the current radio conditions. Similar to cellular networks, the ability to focus or steer RF energy can provide increased throughput in the network and reduced delay via an increase in spatial reuse [12]. These benefits also enable MANETs to be density adaptive. The use of directional antennas on mobile terminals, however, introduces the complex issue of finding the desired direction for transmission or reception. This is most critical in a MANET that does not have centralized control and the terminals may also have limitations with respect to size and the complexity/cost of communication hardware. Transmission range power control can also help to make ad hoc networks density adaptive [13], but new network layer algorithms are needed if the use of power control and beamforming antennas is to be successfully exploited in MANETs.

\subsubsection{Satellite Communications}

The XTAR system is a satellite communications network that is capable of providing tactical aircraft with over-the-horizon communications. XTAR's two-satellite system is based on Space Systems/Loral's space-proven 1300 satellite platform and has been designed 
specifically for US and NATO SATCOM systems [14]. XTAR features two very important aspects in its system. Firstly, it offers flexibility since its spot beams can be dynamically controlled. In addition, XTAR provides larger bandwidth and throughput compared to existing X-band terminals as it has 'stackable' spot beams and also dual polarization capability. The dual polarization feature of XTAR, namely right-hand circular polarization (RHCP) and left-hand circular polarization (LHCP) effectively doubles the spectral efficiency of the system. All of these key elements of the XTAR system make it appear extremely useful for defence-specific communication services.

\subsubsection{Global Positioning Satellite (GPS) System}

The global positioning satellite (GPS) system [15] uses 24 satellites in medium earth orbits to provide accurate position information (latitude, longitude, and elevation) to users on land, in the air, or at sea. Originally developed as the NAVSTAR system by the military at a cost of approximately $\$ 12 \mathrm{~B}$, the GPS has quickly become one of the most pervasive applications of wireless technology for military, consumers, and businesses throughout the world. Today, GPS receivers are found on commercial and private airplanes, boats, and ships as well as ground vehicles. When integrating GPS equipment into vehicles and systems, it is imperative to take into consideration not only parameters such as weight, size, and number of channels when designing the receiver module, but also the jamming susceptibility of the GPS application.

GPSs transmit low-power signals that must travel great distances to reach mobile GPS receivers. In contrast, GPS jamming devices are usually located in relatively close proximity to these GPS receivers and transmit significantly higher power signals. The simplest airborne GPS antenna structures are small single-element omnidirectional radiators. However, the use of omnidirectional antennas makes these devices particularly susceptible to jamming. Techniques such as beam steering and null steering can reduce the effects of jamming. It is well known that one of the most effective means of mitigating the potential issues of jamming is to utilize a controlled reception pattern antenna (CRPA), or, put more simply, a phased/adaptive array. The CRPA is an array of antenna elements that has the ability to modify its radiation pattern for the purpose of rejecting interference signals and/or emphasizing the GPS satellite signal. Present-day CRPAs employ seven or eight elements and are over 13 inches wide in diameter; there is much interest in reducing the overall size of a CRPA without compromising the antenna's efficiency and antijamming capability.

\subsubsection{Wireless Personal Area Network (WPAN)}

Very wide unlicensed frequency ranges in the V-band have been allocated by regulatory agencies around the world, including $57-64 \mathrm{GHz}$ in the US and 59-66 GHz in Japan. Operation of wireless networks in such large frequency bands at such high frequencies enables the transmission of very high data rates. In addition, the high atmospheric absorption due to oxygen that occurs at $60 \mathrm{GHz}$ provides other benefits, such as high security, frequency reuse, and immunity to electromagnetic interference, as highlighted in Figure 1.8. The security features of the $60 \mathrm{GHz}$ transmission band have long been exploited in radio communications for military applications, but more recently it is also being actively pursued for commercial wireless telecommunications. 


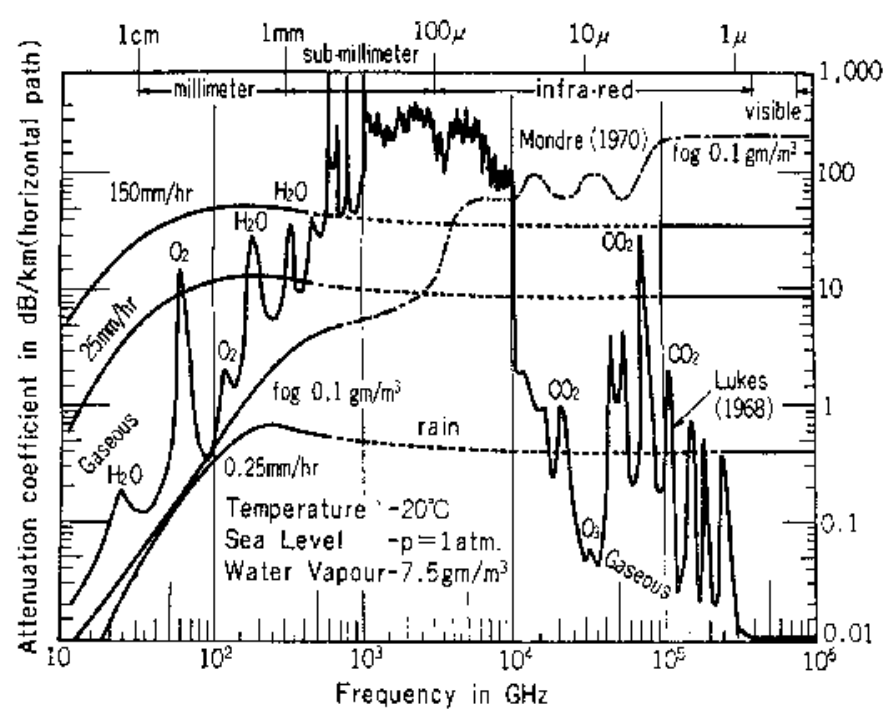

Figure 1.8 Attenuation coefficients of various gases as a function of frequency [19].

While wireless local area network (WLAN) standards such as Bluetooth, IEEE 802.11b, and IEEE $802.11 \mathrm{a} / \mathrm{g}$ have demonstrated a progression in data rate capability from around 1 $\mathrm{Mb} / \mathrm{s}, 11 \mathrm{Mb} / \mathrm{s}$, and $55 \mathrm{Mb} / \mathrm{s}$ [16], respectively, the unlicensed V-band spectrum provides an opportunity to continue the trend for data rate increases, for operation at $\mathrm{Gb} / \mathrm{s}$ rates. As an example, the IEEE 802.15.3 Study Group 3c (SG3c) was formed in March 2004 to explore the use of the $60 \mathrm{GHz}$ band for wireless personal area networks (WPANs) [17]. SG3c is developing a millimetre-wave-based alternative physical layer (PHY) for the existing high data rate IEEE 802.15.3 WPAN Standard 802.15.3-2003 [18]. The millimetre-wave WPAN will allow very high data rate applications such as high-speed internet access, streaming video download, real-time data streaming as well as the wireless databus for cable replacement.

\subsubsection{Combat Identification (CID)}

Combat identification (CID), the ability to differentiate friend or foe with high confidence, is a serious and challenging problem for military forces. Recent military engagements have shown the increased need for forces to be able to identify quickly the presence of their own soldiers within an active battle or combat area. Today, the battles being fought by personnel are increasingly moving from remote combat zones such as rural geographies and jungles to urban and populated centres, where the identification of the presence of friend or foe is particularly problematic. In addition, statistics indicate that approximately $60 \%$ of fratricides historically occur in ground-to-ground scenarios.

Improved CID concepts and technologies can not only increase the effectiveness of military operations but also significantly reduce the number of friendly-fire casualties as well as lower the risk of collateral damage. The focus of this proposed effort is the development of an affordable multimode. Presently deployed CID systems are either inherently expensive, not capable of operating over a wide range conditions or environments, or vulnerable to enemy exploitation. The Individual Combat Identification System (ICIDS) is a dismounted 
soldier CID technology consisting of a weapon-mounted laser interrogator and a helmetmounted radio transponder.

\subsection{NEW WIRELESS PLATFORMS}

Not only are innovative wireless systems being considered to provide new and improved services but new platforms are being developed. Two such platforms are unmanned vehicles and body wearable systems. Both technologies can potentially provide significant advances in wireless communication/sensing services and as a result are receiving a lot of attention from both the military and commercial sectors.

\subsubsection{Unmanned Vehicles}

The role of unmanned vehicles in today's military and first responder sectors is becoming more important. These platforms can be used to provide reconnaissance information without the expense (cost and safety) of a manned mission. An example of this technology is a low-cost, expendable tactical UAV (unmanned aerial vehicle) with interchangeable sensor payloads tailored to the specific operation, which can be launched from another aircraft. There are several types of UAVs and two examples are shown in Figure 1.3.1.

Another example of an unmanned vehicle is the unmanned undersea vehicle (UUV). These self-propelled untethered submersibles operate either autonomously or under minimal supervisory control. Over the last decade, interest in UUVs for commercial applications has increased markedly. These seacraft are receiving wider acceptance in the natural resource and telecommunication industries for monitoring and maintenance of underwater pipes and cables. Oil and gas companies such as Shell now routinely employ UUVs offshore, where they offer particular benefits for offshore oilfield route surveys and pipeline surveillance and repair in deep waters. UUVs are also finding a role in other commercial applications, such as operation in hazardous subsea environments, as well as automated inspections of ship hulls and other infrastructure located underwater. In addition to military applications, other government agencies are considering UUVs for operations such as hazardous waste management (Department of Energy), fisheries research (National Oceanic and Atmospheric Administration), and drug seizures (US Coast Guard). Figure 1.10 shows an example of a UUV.

One of the most critical components of any unmanned vehicle system is the data communication link between the vehicle and the control platform. This data link must be both highly reliable and secure in order to properly control the UAV or UUV and its sensor payload, while also providing real-time jam-resistant transmission of the sensor data. Presently JTRS and even WLAN are being considered for these unmanned vehicles.

\subsubsection{Body Wearable Platforms}

The fact that the current antennas are hindering the ability of soldiers to accomplish their mission due to their unwieldy size helps to substantiate the critical need for antennas integrated into tactical clothing: body wearable solutions. In addition to the military, textile antennas will also find use in the activities of first responders and law enforcement personnel in applications where communication is critical. To achieve interoperability for emergency 


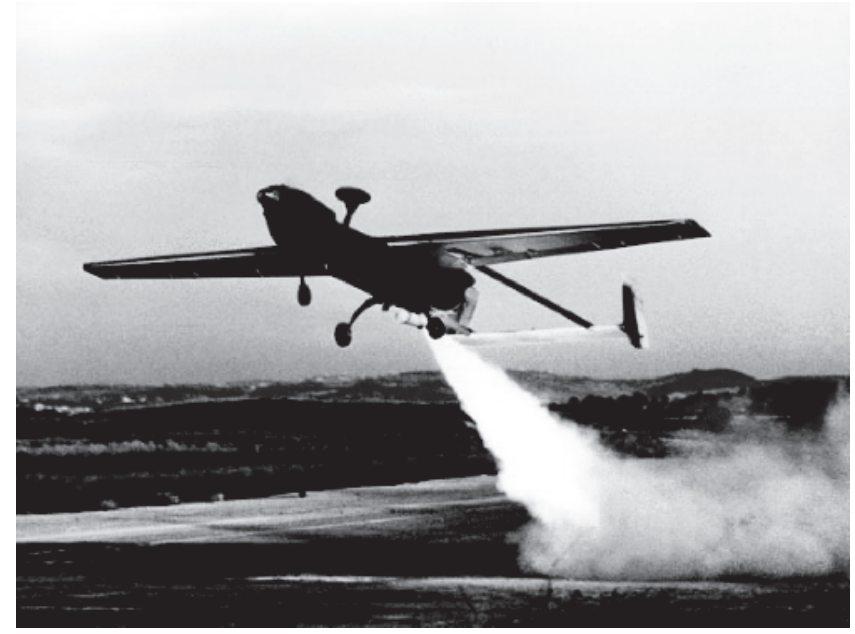

(a)

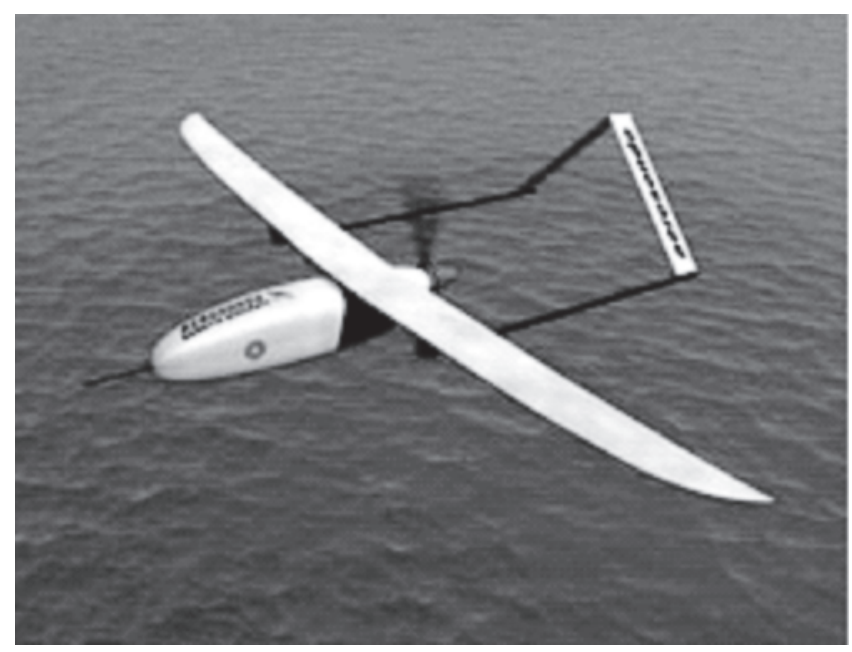

(b)

Figure 1.9 Examples of UAVs: (a) Northrop Grumman's Predator [20] and (b) Aerosonde's $M K I V[21]$.

personnel, first responder voice/data communication equipment is moving to the $700 / 800$ $\mathrm{MHz}$ frequency band and data communication devices operating within the IEEE 802.11 WLAN 2.4-2.5 GHz band continue to proliferate. The ability to provide firefighters with the capability to carry out hands-free communications without the need for obtrusive antennas helps to realize a safer communication system for such emergency personnel. In general, the elimination of obtrusive antennas either through helmet-mounted antenna technology or integrated uniform antenna solutions, will aid many first responders. An example of a body wearable antenna integrated within a tactical vest is shown in Figure 1.11. 


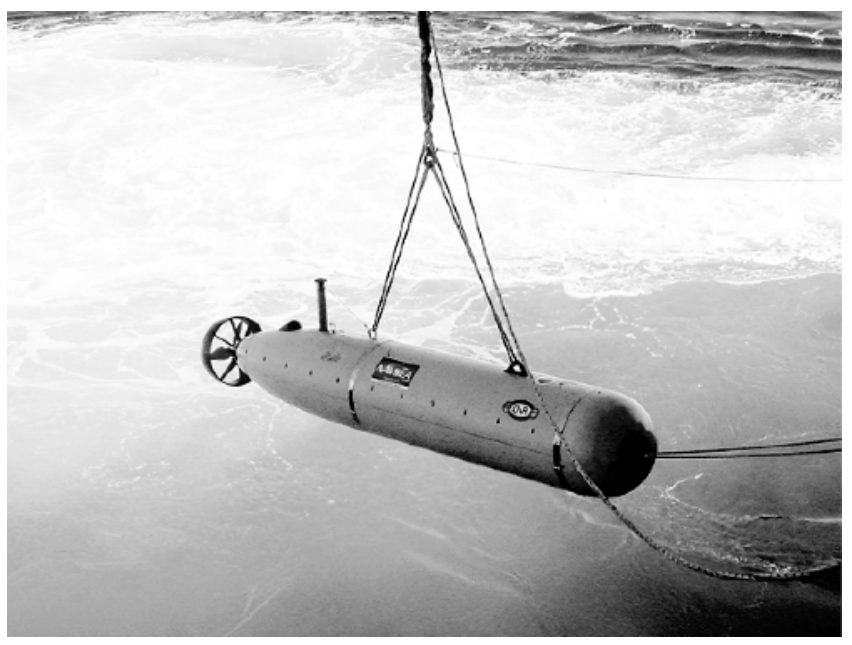

Figure 1.10 A UUV developed by Bluefin [22].

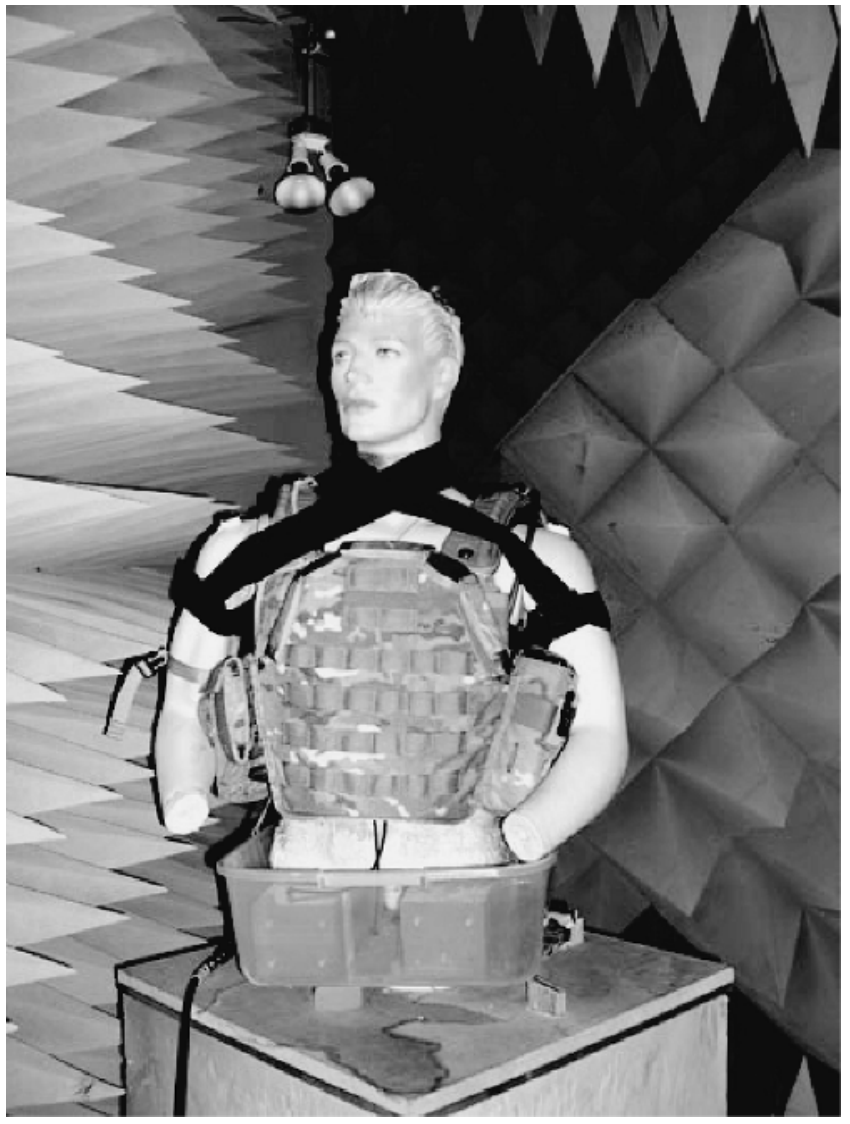

Figure 1.11 Photograph showing body wearable antennas integrated in a combat vest. 


\subsection{ANTENNA REQUIREMENTS}

As should be apparent from the previous examples of wireless systems being considered for both the military and the commercial markets, there is a wide range of antennas needed to interface with these systems; that is a 'one antenna' solution is not realistic. This is why this book comprises more than one chapter! There are, however, some fundamental properties that the antenna engineer must address to ensure the chosen solution operates in the best possible manner, for a given volume. Of course these characteristics are not independent and of course the engineer typically must compromise between the parameters to give the optimum result.

\subsubsection{Size}

The size (volume) of the antenna and its overall impact on the surrounding environment is extremely important for most wireless communication systems. Not only does the antenna need to be unobtrusive and extremely small but it is typically desired that its performance be equivalent to an antenna developed in isolation. There are of course limits to how small an antenna can be, that is to say if an efficient solution is required. Having said this, there are technologies that can be used to reduce the size of an antenna effectively and some of these are investigated in later chapters. However, associated with most of these procedures is a compromise in performance, typically bandwidth (return loss bandwidth) and gain. Also, as a general rule of thumb, the more narrowband the solution the more susceptible it is to its surrounding environment. Fortunately a lot of communication systems (such as WLAN) do not require large instantaneous bandwidths and therefore this is not an issue. Also there are a lot of sophisticated electromagnetic (EM) software packages available that can accurately model the surrounding environment of an antenna and so variations in the performance due to, say, the packaging can be taken into account.

One point that should be made clear here is that the size of the antenna includes the ground-plane used to create the radiator. Unfortunately there has been a plethora of 'small' antennas that have recently been proposed in the literature that actually have large groundplanes. The fact is that if the antenna requires a large ground-plane to operate, then the size of the antenna is set by the size of that ground-plane.

Probably the biggest issue with utilizing small antennas for wireless communications is the reduction in efficiency. Effectively, the lower the efficiency, the more power is required from the front-end RF amplifiers and therefore the lower the battery life of the wireless terminal (if it is a terminal under consideration). Despite this, advances in efficient RF amplifiers and new battery technology are helping to resolve this potential problem.

\subsubsection{Integration}

The ease of integration is a very important consideration in antenna technology. Radiators that can be easily integrated with the RF front-end are overall cheaper solutions. Typically, too, the easier the integration the more efficient the solution, which is very important for wireless applications in the high microwave frequency bands. Integration does not only refer to the RF engineering, but also the structural properties. For example, in the UAV applications, it is imperative the antennas used can reside within the surface of the platform 
such that the aerodynamics of the vehicle is not greatly impacted. Of course the 'integrated' radiator cannot adversely affect the structural integrity of the vehicle. This is why appliqué antennas are receiving considerable attention today.

\subsubsection{Efficiency}

As mentioned before, efficiency is very important for any radiator; the greater the efficiency the better the link budget. Other than the size of the radiator, another factor that impacts the efficiency of the antenna is the material used to create the structure, or surround the radiator. For printed antennas, the supporting substrate/dielectric laminate needs to be low in loss, particularly for high-frequency applications. Although FR4 is a low-cost readily available laminate, for frequencies above $2 \mathrm{GHz}$ the dielectric losses significantly reduce the overall efficiency of the radiator, making this a cheap but unattractive solution. High dielectric constant ceramics are becoming more abundant and due to the dielectric loading effect of the material on the radiator, small solutions can be developed. Care must be taken to ensure that the high dielectric constant material is low loss, as typically the loss tangents of these materials are higher than their low dielectric constant counterparts and therefore the overall impact on the radiation performance can be severe.

As there are many antenna solutions for wireless applications, there is a common quandary often considered: should I choose a wideband solution or one that just meets the bandwidth requirements? In terms of efficiency, if low-loss material is used typically a narrowband solution will be smaller and also more efficient over the desired wireless band than a wideband radiator. Having said this, a wideband solution is typically more 'forgiving' of fabrication tolerances, although more dependent on its surrounding environment.

\subsubsection{Bandwidth}

The designed antenna must satisfy the bandwidth requirements for the wireless system. Of course there are two fundamental bandwidth criteria the antenna must typically meet: the impedance bandwidth (return loss bandwidth) and the gain bandwidth. There are no universal definitions of these bandwidths that apply to every wireless system, but typically the return loss bandwidth is the frequency range in which the return loss is better than -10 $\mathrm{dB}$, which means that less than $10 \%$ of the power is reflected into the RF circuitry. There have been cases where $-6 \mathrm{~dB}$ has been acceptable for an antenna, due to the stringent size constraints placed on the radiator. However, there are other systems where $-20 \mathrm{~dB}$ is required, in particular if 'ghosting' is an issue. The gain bandwidth is usually less than a 3 $\mathrm{dB}$ variation across the range of frequencies, although for some systems a $1 \mathrm{~dB}$ variation is more appropriate. For narrowband wireless systems, the gain requirement is typically not as hard to meet compared to the return loss bandwidth for a constrained volume.

There have been some alarming trends noticed in the literature, in particular with the development of wideband printed antennas; researchers tend to focus on the return loss bandwidth of their proposed antenna, without considering the gain bandwidth. This could be a consequence of two factors: history and ease. Historically, when one of the most prevalent printed antennas, the patch antenna, was investigated, because of its inherent narrowband nature, the impedance variation was more of an issue than the gain variation of the antenna. In fact in its basic form the return loss bandwidth of a patch antenna is smaller than the gain 
bandwidth by a factor of 10 or so. For this reason, much research was dedicated towards accurately modelling this form of radiator and also the enhancement of the return loss bandwidth. Unfortunately in the process it appears that some of the more recent researchers have forgotten about the gain response, which is equally as important, if not more so. This is especially true when investigating radiators with bandwidths in excess of several octaves. It should always be remembered that a matched load has an excellent return loss bandwidth, but does not radiate! The other reason for this trend may be associated with the cost of making the appropriate measurements. Return loss measurements are relatively straightforward to make using a vector network analyser, whereas gain measurements need some form of antenna range, whether it be an anechoic chamber, near-field facility, or outdoor range. These facilities are expensive and perhaps not accessible to most researchers.

\subsubsection{Polarization}

The polarization of an antenna is an interesting issue and in some wireless system cases its investigation and consequences have become a 'lost art'. Satellite communications have always had well-defined polarization requirements, but terrestrial systems have really been a case-by-case scenario and it really depends on what part of the link is being designed. For example, most base stations in present-day cellular systems use dual linear polarization to take advantage of multipath effects and also the different propagation effects on horizontal and vertical polarization, whereas the handset terminal requirement is typically 'whatever you can get' simply to the restricted size constraints and therefore there is great difficulty in controlling the polarization.

In general most wireless systems prefer some form of polarization diversity, whether it be to reduce the probability of 'drop-outs' or to increase the capacity of the system. The latter requires strict polarization control.

\subsubsection{Power Handling}

The power handling requirements for an antenna once again really depend on the application, with some radar systems requiring greater than $100 \mathrm{~kW}$ capability. Most wireless systems do not need such large power handling requirements and typically maxima in the order of 1-10 $\mathrm{W}$ are acceptable. The power handling capability of an antenna is fundamentally determined by the materials used to develop the radiator. Having said this, certain classes of radiators can handle high power levels better than others; for example waveguide-based radiators can operate efficiently at very high power levels. For base station antennas where the power handling requirements are higher, to avoid arcing and other high power level effects, the number of solder joints and other potential current discontinuities should be minimized.

\subsubsection{Summary}

Looking at these generalized requirements and also the need for low-cost solutions, printed antennas would appear to be the best choice of radiator. Of course printed antennas come in many forms and so the more appropriate choice for a particular application may not be obvious. The reader should keep in mind that having made such a statement, there will 
always be applications and the need for conventional antennas: waveguide-based, reflectors, and wire-based. In fact a lot of the printed antennas presented in the upcoming chapters have their origins in nonprinted antenna technology or can easily be derived from them. Several obvious examples are the shorted patch/planar inverted-F antenna (PIFA) and its derivation from the F-type wire antenna created in the 1940s. Also, the relationship between the printed reflectarray and the classic reflector, as well as the printed quasi Yagi Uda and its wire predecessor, seem quite obvious. Maybe not such an obvious link is the tapered slot antenna and the horn waveguide radiator, although close examination of the characteristics and design trends associated with both of these radiators do imply a strong connection.

\subsection{FUNDAMENTAL PRINTED ANTENNAS}

In this section, an attempt will be made to summarize the many forms of printed antennas. Most of these radiators will be discussed in more detail in subsequent chapters. A decision was made to divide printed antennas further into three categories: inherently wideband radiators; resonant style antennas; and small radiators. Of course these radiators are not totally independent; for example it is possible to develop a narrowband version of one of the wideband solutions and conversely by using multiple narrowband radiators a wideband element can be designed. Thus the divisions used here are somewhat grey.

\subsubsection{Wideband Printed Antennas}

Inherently wideband printed antennas are typically derived from travelling wave antenna concepts. From the IEEE Standard Definitions of Terms for Antennas (IEEE Std 145-1983) [23], travelling wave antennas are defined as 'an antenna whose excitation has a quasiuniform progressive phase, as a result of a single feeding wave traversing its length in one direction only'. Thus any antenna that relies on this mechanism to radiate falls into this class, and as can be imagined there is a plethora of antennas that do so. Examples include beverage antennas, horn antennas, reflectors, tapered slot antennas, and lens antennas. A more simplified interpretation of the above IEEE standard definition, which is not necessarily valid for all forms of travelling wave antennas, is that this form of radiator converts guided electromagnetic energy into radiated energy in a gradual or smooth transition. An example of a travelling wave antenna, whose form is obviously compliant with this statement, is a horn antenna where the flange of the waveguide is gradually opened to match effectively the impedance/mode of the guided wave to that of free space. As stated before, travelling wave antennas are not necessarily wideband in nature, but because of the smooth transition from the guided medium to the unguided medium they tend to be, at least with careful design.

\subsubsection{Tapered Slot Antennas}

The tapered slot antenna is undoubtedly the most common printed travelling wave antenna (see, for examples, References [24] to [27]). These antennas can operate over multioctave bandwidths and can operate well in a phased array environment. Effectively this form of printed radiator can be thought of in the same manner as a horn antenna, where a 


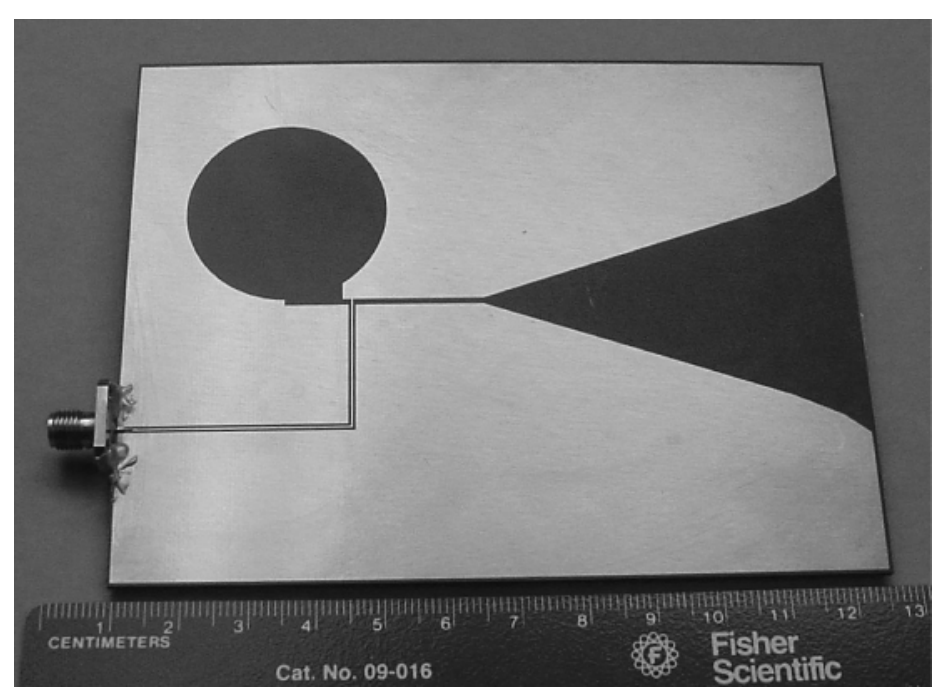

Figure 1.12 Photograph of a tapered slot.

waveguide is flared out to 'match' the electromagnetic wave to free space, although in this case the transmission medium is a slot line. However, unlike a conventional horn antenna the fundamental mode of the slot line is transverse electromagnetic (TEM) in nature and so some of the issues associated with the wave matching of a horn antenna are mitigated here. Having said that, the tapered slot is a relatively complicated radiating element with many degrees of freedom. As is the case for a horn antenna, the profile of the taper (or flare) contributes to the input impedance behaviour as well as the radiation performance of this printed travelling wave antenna. Common profiles include linear, exponential, and piecewise linear. One particular challenge with a tapered slot antenna is the transition from the slot line to a more common transmission media used in microwave circuitry design.

Figure 1.12 shows a photograph of a tapered slot antenna with a coplanar waveguide (CPW) feed [28]. Incorporated in this design is a wideband balun (part of which is the large slot-line cavity in the top left-hand corner of the photograph), which efficiently transfers energy from an unbalanced transmission line (CPW) to the balanced slot-line. Figure 1.13 shows the predicted and measured return loss of the developed travelling antenna, as well as a typical radiation pattern.

\subsubsection{Printed Spiral Antennas}

Spiral-shaped antennas are well known to give very wideband impedance and radiation responses [29]. In their traditional form these antennas radiated equally in the planes both above and below the spiral radiator (whether in the form of a wire/conductor or a slot). To create a unidirectional radiation pattern, spirals were loaded by a lossy cavity. A consequence of such a loading was that the volume of the antenna increased dramatically as the cavity depth had to be greater than the radius of the spiral, and therefore it was more challenging to integrate these radiators into the surfaces of potential platforms. 


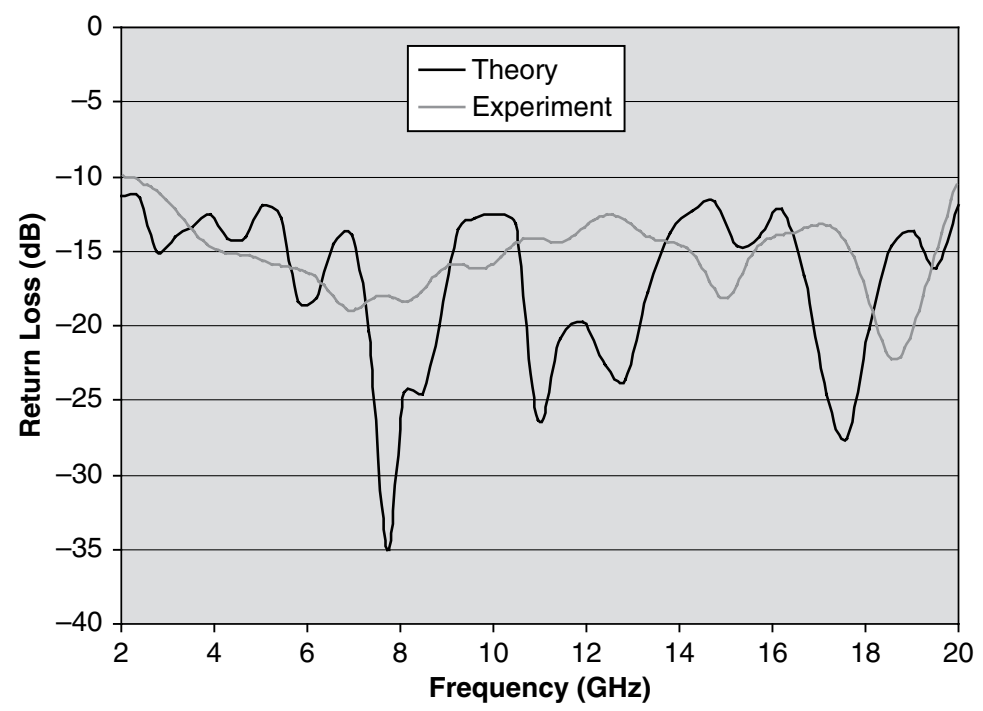

(a)

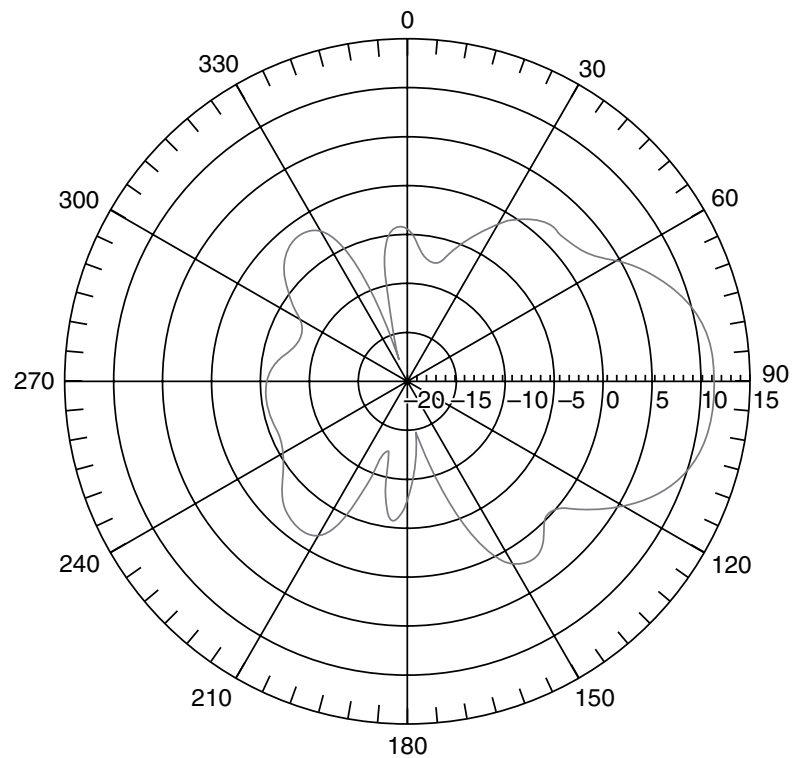

(b)

Figure 1.13 Performance of the tapered slot of Figure 1.12: (a) return loss and (b) radiation patterns.

In the early 1990s, a low-profile spiral was developed that significantly reduced the depth of the antenna [30]. Here a wideband printed spiral antenna was developed using a grounded laminate of thickness comparable to that used for microstrip patch antennas over the same frequency range. The key to obtaining a good impedance response and radiation performance from this low-profile spiral was to incorporate an absorbing material around the perimeter of the spiral conductor and also include the design of a wideband balun. A simpler version of this approach is shown schematically in Figure 1.14. Here one arm of the spiral 


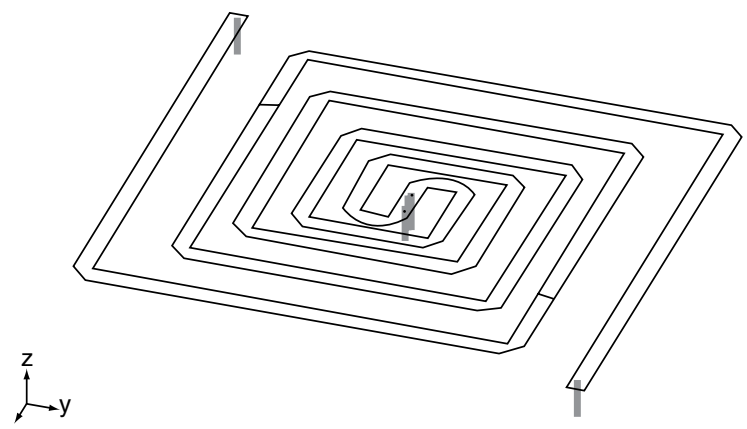

(a)

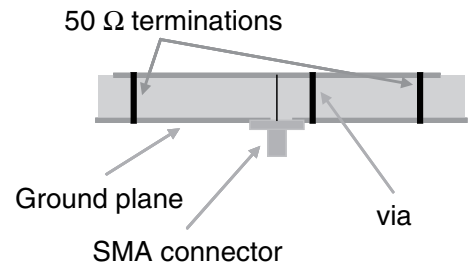

(b)

Figure 1.14 Schematic of a simplified printed spiral antenna: (a) three-dimensional view and (b) cross-sectional view.

is probe fed, while a via is used to connect the other arm of the spiral to the ground-plane below the printed conductor. The arms are $50 \Omega$ microstrip tracks and are terminated with $50 \Omega$ resistors. Not using a balun can give responsible performance as the thickness of the dielectric layer is less than $0.05 \lambda_{0}$ (where $\lambda_{0}$ is the free-space wavelength at the highest frequency of operation). As expected, the return loss performance of this simplified printed spiral antenna degrades at higher frequencies because of the oversimplified feed structure. Of course spiral antennas loaded with resistive elements do have lower radiation efficiencies than nonloaded radiators (such as tapered slots).

Figure 1.15 shows the predicted return loss performance of the printed spiral shown in Figure 1.14. As can be seen from Figure 1.14, the return loss is better than $-10 \mathrm{~dB}$ over a wideband bandwidth and as the frequency increases the return loss performance degrades. Figure 1.16 shows the predicted radiation patterns of the printed spiral at $1 \mathrm{GHz}$.

\subsubsection{Resonant-Style Printed Antennas}

Although the introduction of 'wideband' wireless systems (for example JTRS) would imply that inherently narrowband technologies are no longer relevant, this is not the case. A key factor is the definition of 'wideband', and it seems that some systems have 'stretched' the definition perhaps due to the marketing potential of the phrase or perhaps to differentiate the proposed technology from previous generations. For example, some of the cellular systems near $2 \mathrm{GHz}$ were touted as being wideband because the user bandwidth was more than the $900 \mathrm{MHz}$ systems. This statement is true in a relative sense, relative to previous generations 


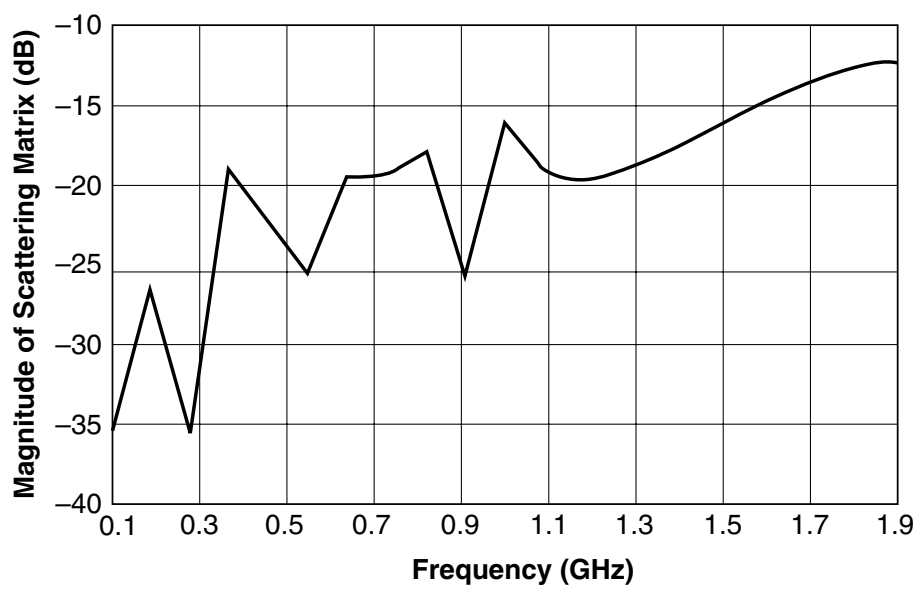

Figure 1.15 Return loss performance of the spiral antenna presented in Figure 1.14.

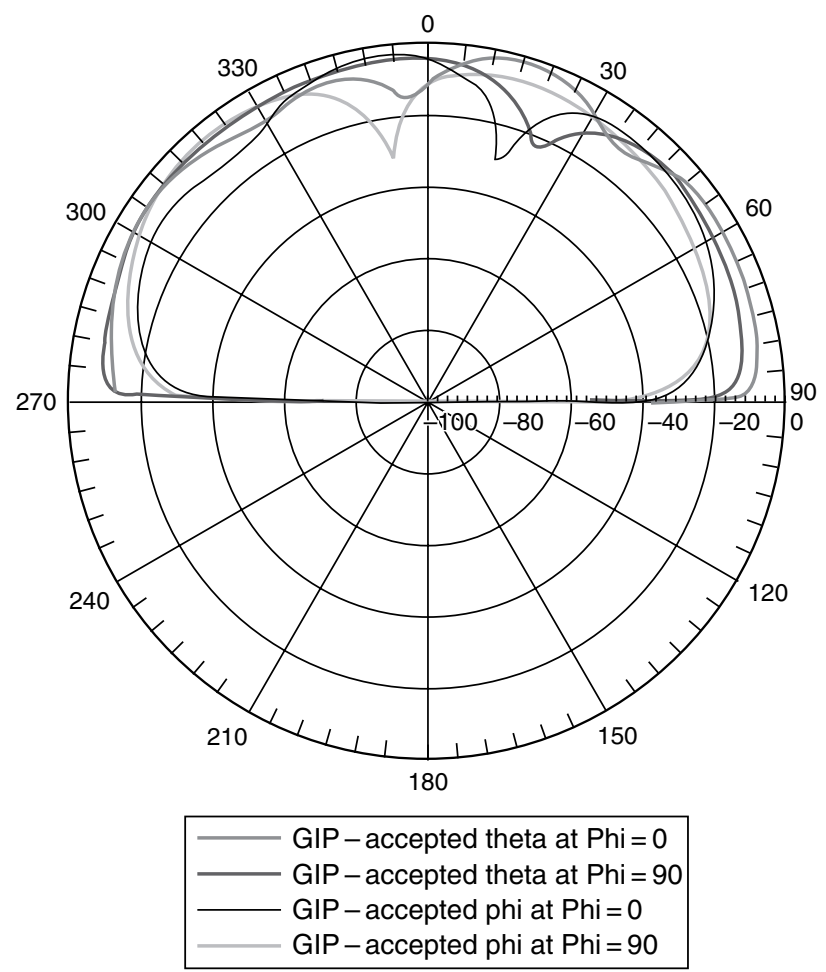

Figure 1.16 Typical radiation patterns of the spiral antenna presented in Figure 1.14.

not frequency; the bandwidth is more than the previous generations, but with respect to the frequency of operation the increase in bandwidth is minor and could hardly be deemed wideband. Having said this, there are some truly wideband applications, such as JTRS or UWB, and there are multiband systems too, of which an option may be just to utilize a wideband radiator. 


\section{WIRELESS SYSTEMS AND PRINTED ANTENNAS}

From a designer's prospective, when would using a resonant-style radiator be considered and why would narrowband technology be considered when wideband radiators can easily accommodate the electrical requirements? Resonant-style radiators do have several antennas over their travelling wave counterparts. If the bandwidth is small enough, resonant-style antennas tend to be more efficient (if low-loss materials are used). Resonant-style antennas tend to be smaller in size (typically less than $\lambda_{\mathrm{g}} / 2$, where $\lambda_{\mathrm{g}}$ is the guided wavelength), which can allow for arrays of these elements to be formed readily with therefore good control of the radiation performance. Resonant-style antennas, because of their inherent narrowband nature, can help with out-of-band rejection of noise and therefore assist the overall RF performance of the link, although the first two reasons are probably the most important advantages of resonant-style antennas.

The three fundamental elements for printed resonant-style antennas are: the dipole, the slot, and the patch. Although there have been many variations of these printed radiators developed over the years, the principles of operation of the 'new' versions can be traced back to these three fundamental radiators. Feeding or coupling power to and from these antennas plays a critical role in the overall performance of the antenna, as is the case for any radiator, but it is probably more correct to say that the feeding process, if done properly, allows advantage to be taken fully of the inherent properties of the antenna.

As mentioned before, there is definitely a 'grey' zone when addressing wideband and narrowband antenna technologies. A good example is the aperture stacked patch [31], of which a photograph of this multilayer/element radiator with the layers separated and sandwiched together is shown in Figure 1.17. Here the antenna is developed by combining three 'narrowband' elements (two patches and one slot). The resulting radiator can have bandwidths in excess of an octave, thereby perhaps qualifying it as a wideband radiator. Another example of a wideband antenna developed from narrowband elements is the log-periodic antenna. Once again, the overall response of the antenna is wideband in nature but the individual elements are not.

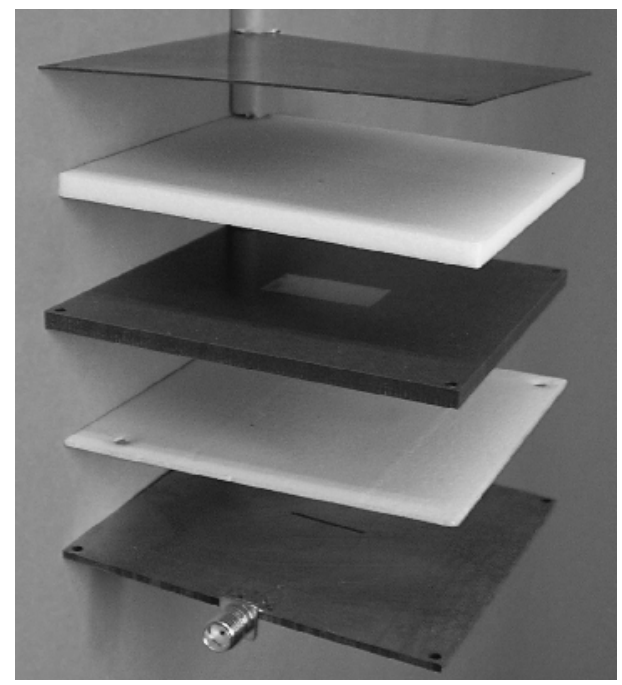

Figure 1.17 Photograph of an aperture stacked patch. 

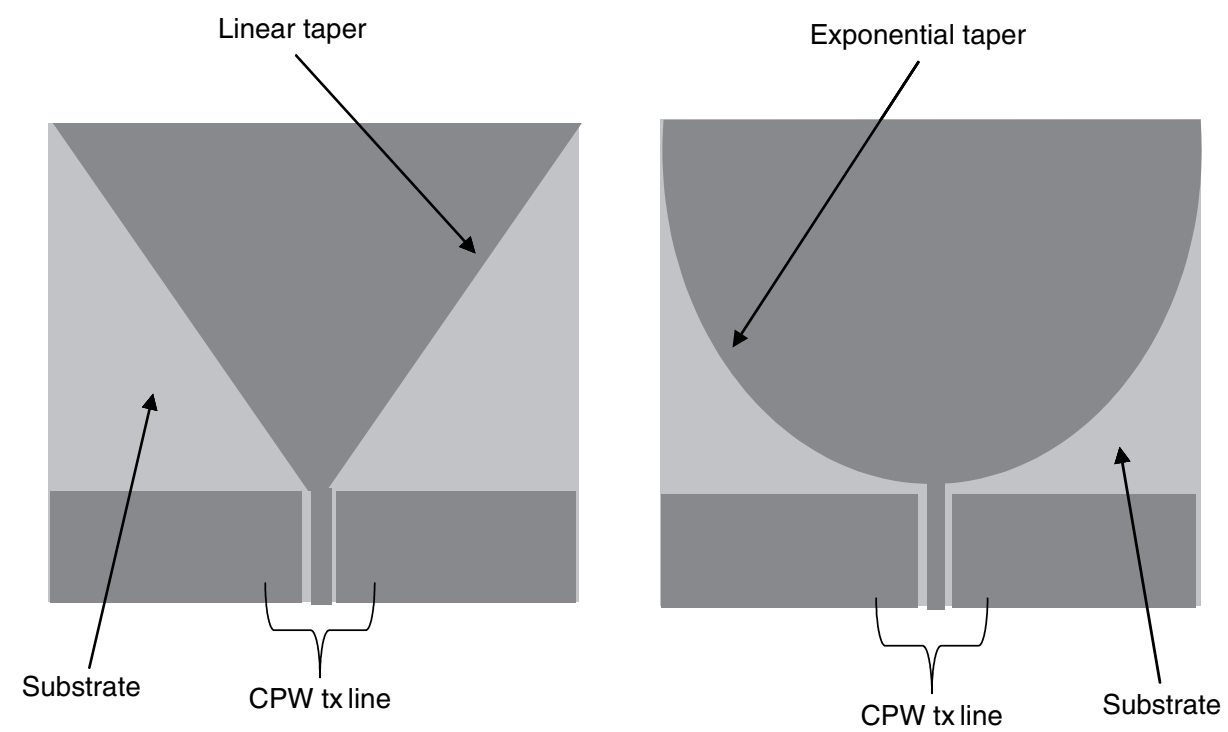

Figure 1.18 Schematics of profile optimized monopoles.

To complicate this issue further, several wideband printed antennas have recently been reported, which can be considered as variations of these narrowband counterparts. In particular, a class of printed dipoles, monopoles, and slots can be considered as 'profile optimized' versions, of which a 'bow-tie' dipole is a well-known example. Figure 1.18 shows schematics of some CPW-fed 'profile optimized' printed monopoles. Here effectively the shape of the conductor (or aperture for the slot case) has been modified to improve the impedance matching response of the antenna, in a similar vain to many travelling wave antennas, such as a tapered slot. These antennas can operate relatively efficiently over multioctaves but it can be argued that their origins stem from narrowband alternatives.

\subsubsection{Printed Dipoles}

Figure 1.19 shows a schematic of a printed dipole antenna, one of the fundamental printed resonant-style radiators. Here the balanced feed, in this case the coplanar stripline (CPS), is used to couple power to and from the resonant antenna. Usually a thin dielectric laminate is used to develop the printed feed and dipole. Figure 1.20 shows the predicted return loss performance and radiation patterns (at the centre of the matched band) of a typical printed dipole. One issue associated with a dipole solution is the sensitivity of the radiator to the presence of a ground-plane, which is hard to avoid in most communication platforms. For this reason, monopoles are a much more attractive radiator solution.

\subsubsection{Printed Slots}

Printed slots are the magnet equivalent of a printed dipole radiator. Because of the presence of the inherent ground-plane these printed antennas are more common than their dipole counterparts. Figure 1.21 shows a schematic of a folded printed slot antenna fed by a 


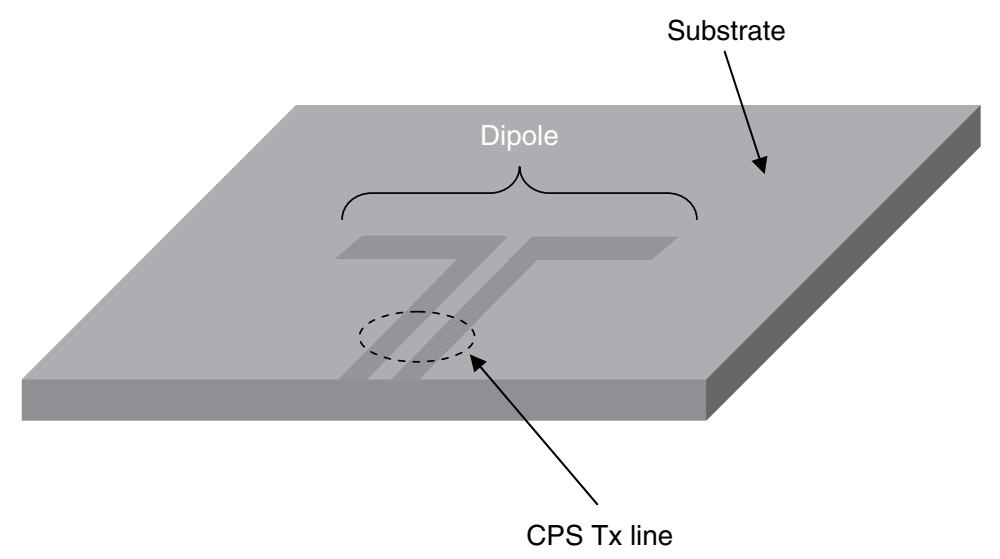

Figure 1.19 Schematic of a printed dipole.

microstrip transmission line. Unlike a dipole, a balanced transmission line is not required to feed this form of printed antenna. The printed slot is folded to ensure a good impedance match at the designed frequency; the number of folds depends on the dielectric constant of the material used to fabricate the antenna [32]. The width of the slot is typically very narrow $\left(0.01 \lambda_{\mathrm{g}}\right)$, although the bandwidth of the antenna can be enhanced by making it larger [33].

Figure 1.22 shows the predicted return loss performance and radiation patterns of a printed folded slot antenna. For this case considered, the ground-plane is assumed infinite in extent.

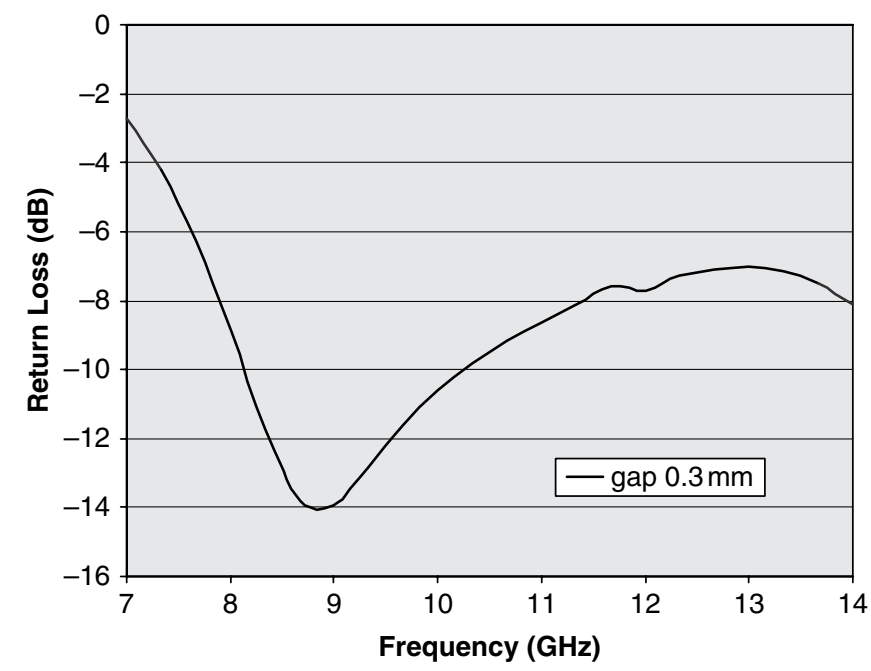

(a)

Figure 1.20 Predicted performance of a printed dipole: (a) return loss and (b) radiation patterns. 


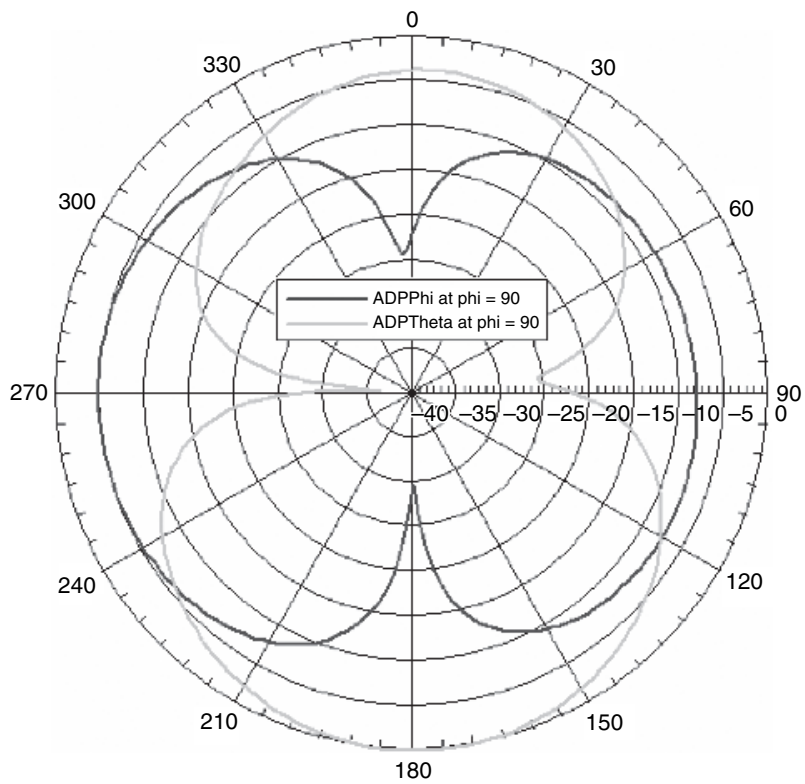

(b)

Figure 1.20 (Continued).

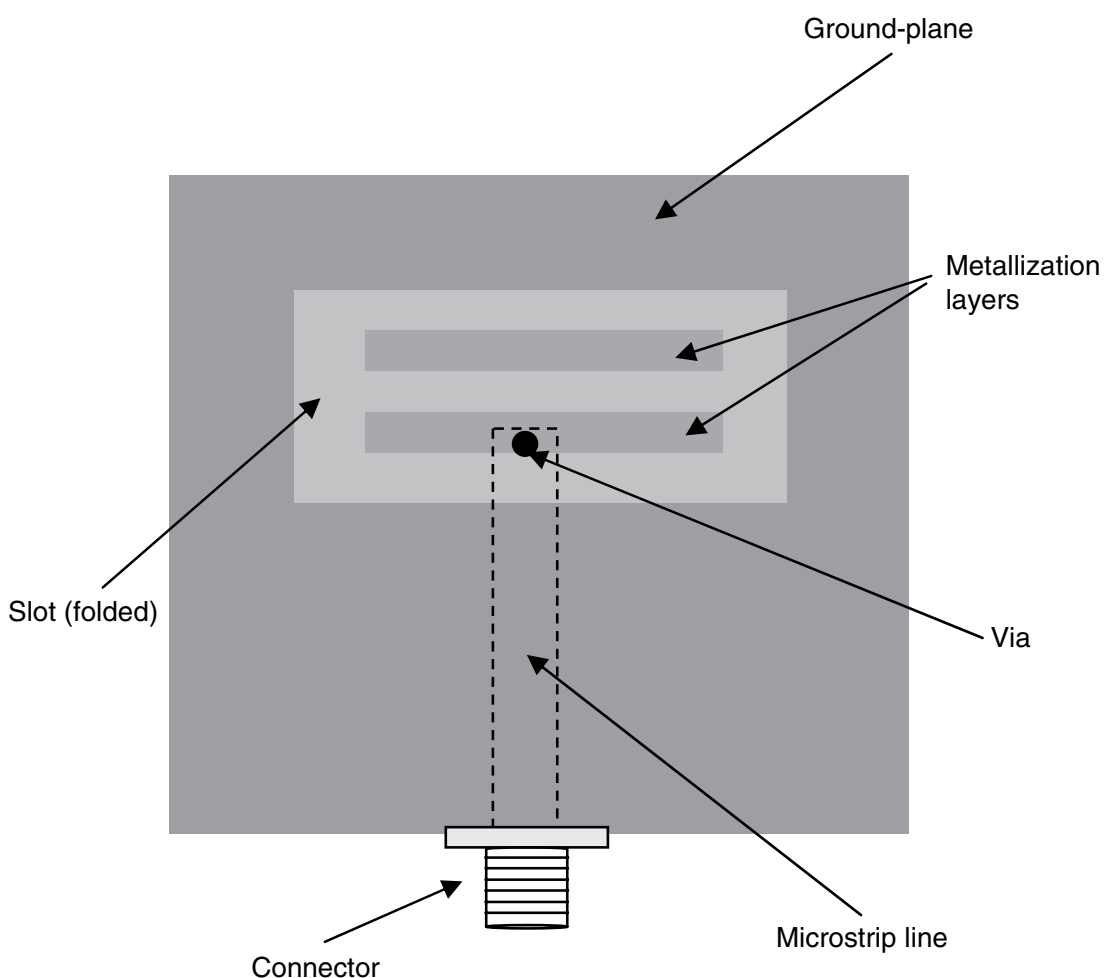

Figure 1.21 Schematic of a microstrip feed folded slot. 


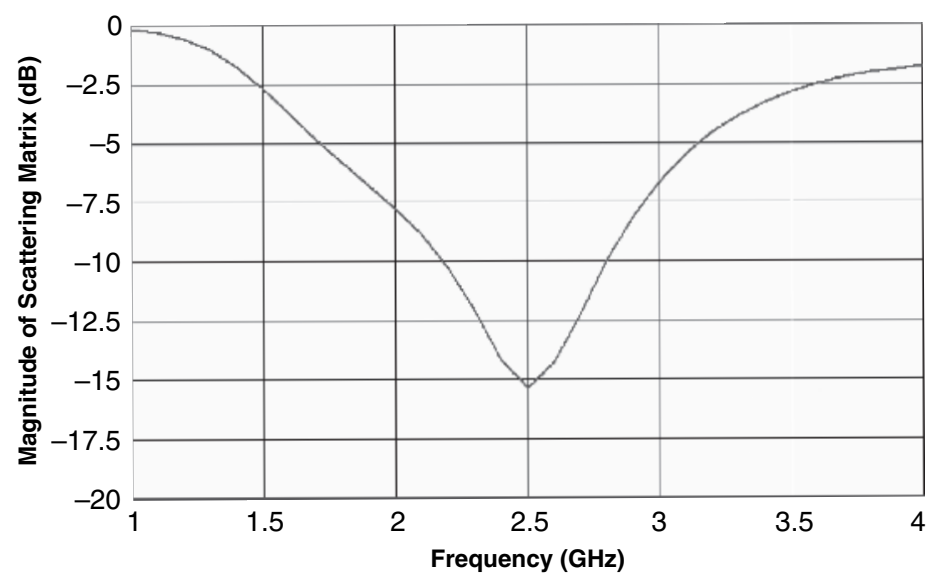

(a)

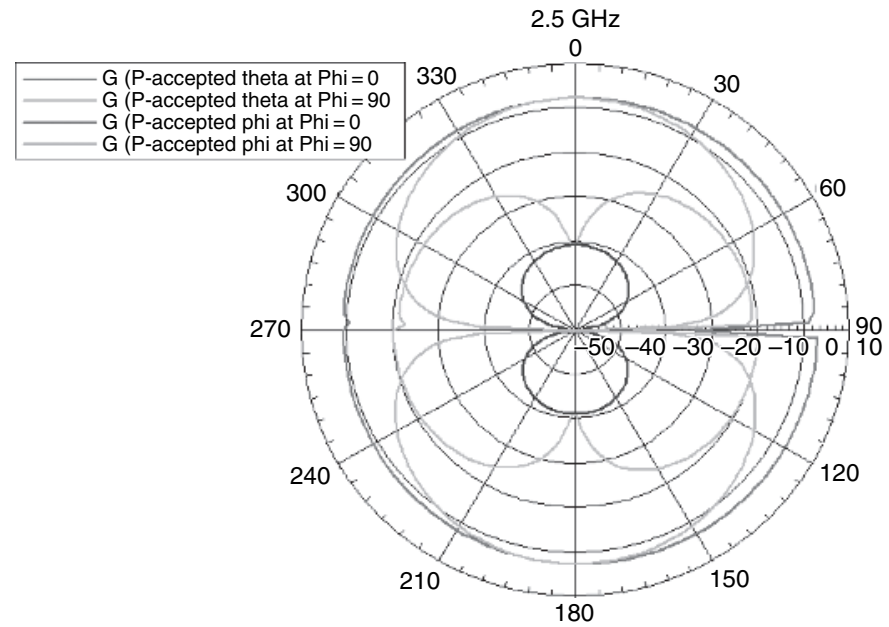

(b)

Figure 1.22 Performance of a printed slot antenna: (a) return loss and (b) radiation patterns.

\subsubsection{Microstrip Patches}

Microstrip patch antennas are a very common printed resonant-style radiator and Figure 1.23 shows a probe-fed example of one. Here a rectangular-shaped patch etched on a grounded dielectric material is fed by a probe that rises through the grounded substrate and is soldered to the patch conductor. Microstrip patches have several advantages compared to printed dipoles and slots and probably the most important is their unidirectional nature (and consequently higher gain) because of their ground-plane. It is this ground-plane that unfortunately limits the bandwidth of the antenna; of the three fundamental printed resonant-style radiators, it probably has the smallest bandwidth.

Like the slot and dipole, there have been many versions of patch antennas examined over the years, including different-shaped patches and varieties of feeding procedures. As mentioned 


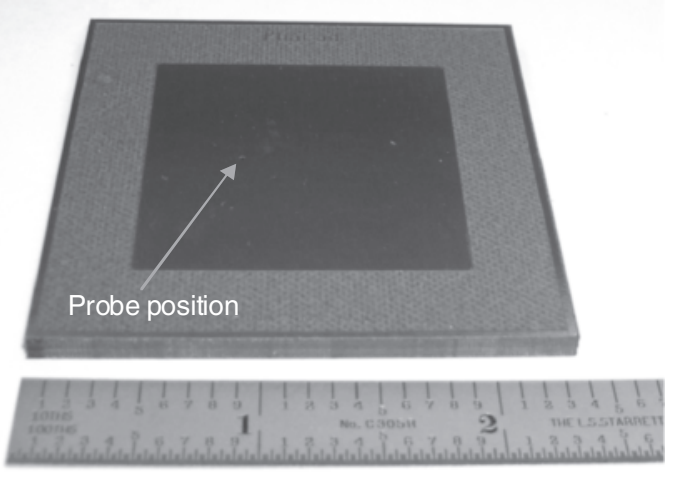

Figure 1.23 Photograph of a probe-fed microstrip patch antenna.

before, the method of feeding an antenna can prove beneficial in terms of optimizing the overall performance, both return loss and radiation patterns, of the radiator. For microstrip patches the two general classes of feeding procedures can be divided into direct contact, where the feed electrode comes into contact with the patch radiator, and noncontact, where the feed line is parasitically coupled to the antenna. Each method has its relative merits and disadvantages and a detailed discussion on these can be found in Reference [34].

Figure 1.24 shows the return loss performance of a typical microstrip patch antenna as well as the predicted radiation patterns at the centre of the $-10 \mathrm{~dB}$ return loss band. For the case considered here, the ground-plane is assumed infinite.

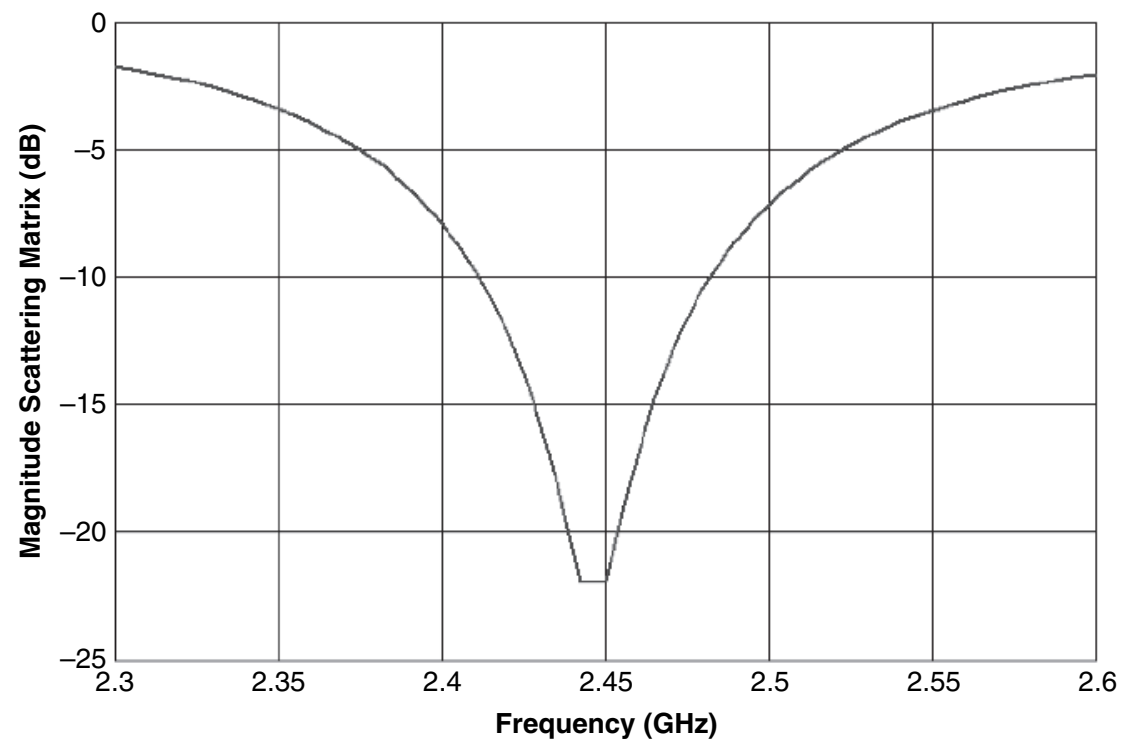

(a)

Figure 1.24 Performance of a microstrip patch antenna: (a) return loss and (b) radiation patterns. 


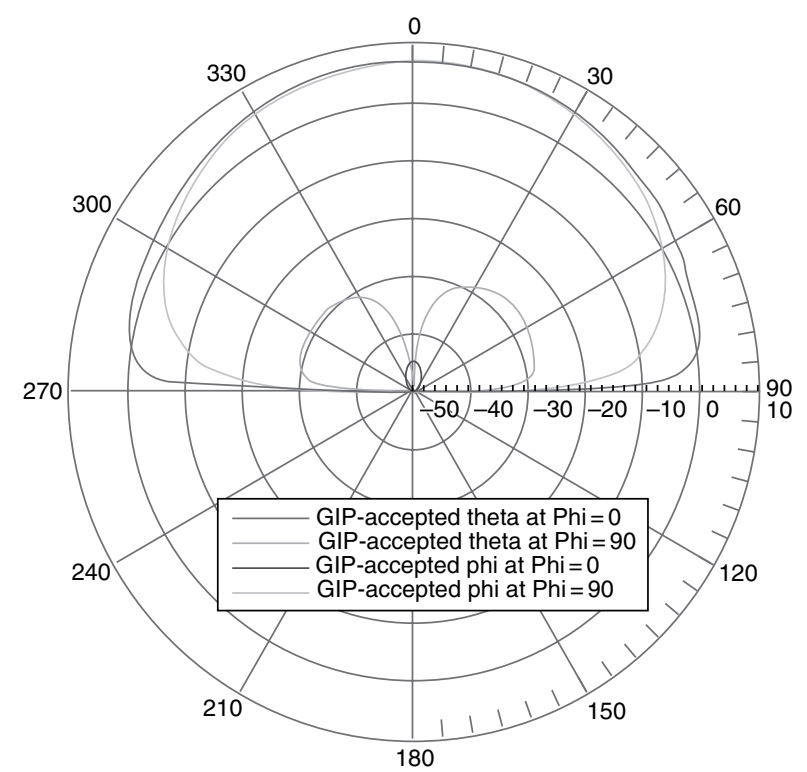

(b)

Figure 1.24 (Continued).

\subsubsection{Small Printed Antennas}

It is because of the requirements for small antennas in wireless terminals (in particular handsets) that there has been considerable research and development undertaken in the area of small printed antennas over the years. Researchers throughout the world have taken the fundamental resonant-style printed antennas and applied size-reduction procedures to yield antennas that can reside in small volumes. Of course this is one of the most difficult challenges an antenna engineer can face, namely to develop an electrically small radiator that can satisfy the radiation performance requirements of most wireless systems. Fortunately there have been significant advancements made in other EM aspects of engineering that has helped the designer, in particular the development of sophisticated electromagnetic software tools that can model not only the radiator but the surrounding environment. These software tools tend to be computationally intensive and therefore the advancements made in computing power cannot be ignored, which has also helped the antenna engineer to use these software packages as design tools (not just as an analysis engine). Having said this, there are compromises that are made when making an antenna small, and it would be almost irresponsible to ignore them. Bandwidth and gain of any antenna are related to its volume and therefore a smaller antenna will have lower gain and smaller bandwidth. Once again, through advancements made in other aspects of engineering, this impact on the overall performance of the wireless system can be minimized. For example, higher quality RF amplifiers (in terms of efficiency and cost) are being developed that ease the gain and return loss requirements of the small antenna. It is interesting to note that most commercially available antennas for cellular handset terminals quote a $-6 \mathrm{~dB}$ return loss bandwidth and have gains of less than $0 \mathrm{dBi}$. It can be extrapolated that this is because the requirements of $-10 \mathrm{~dB}$ return loss and gains greater 
than $0 \mathrm{dBi}$ are just too difficult to achieve for such constrained volumes, and other parts in the system (including the amplifiers) can be used to offset the degradation in the system performance.

There are a variety of procedures that have been successfully employed to reduce the overall size of a printed antenna. These include dielectric loading (both permittivity and permability); using shorting posts (both shorted patches and planar inverted-F antennas, PIFAs); fractal engineering; and maximizing the radiator size within the volume allocated for the antenna, or three-dimensional antenna engineering. Some of these topics will be discussed in later chapters.

Figures 1.25 to 1.28 show four examples of how small versions of the fundamental printed resonant-style radiators can be developed. In Figure 1.25 a CPW-fed printed monopole is shown where the monopole conductor meanders back and forth in order to maximize the area for the given radiator effectively [35]. Figure 1.26 shows a three-dimensional version of a flared (or triangular-shaped) monopole, where the conductor is folded over the edge of the supporting substrate to increase the length of the antenna effectively and subsequently reduction of the operation frequency is realized [36]. Figure 1.27 shows a photograph of a shorted patch antenna. Here shorting pins are used to reduce the size of the patch conductor. Two pins are used to increase the separation distance between the shorting points (the pins) and the probe feed [37].

In Figure 1.28 three concepts are combined to yield a small version of a slot radiator: (1) dielectric loading; (2) folding the slot; and (3) using a short-circuit [38]. Of course there are many more procedures that can be used to reduce the size of the antenna, and judging by

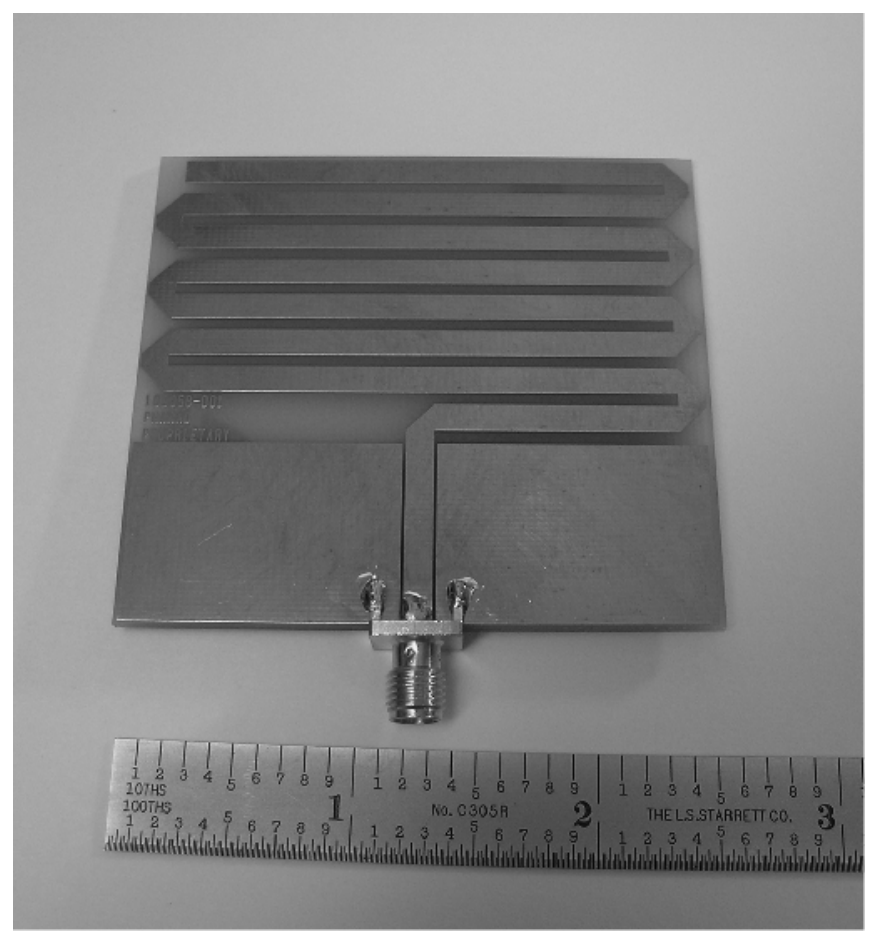

Figure 1.25 Photograph of a printed meander line monopole. 


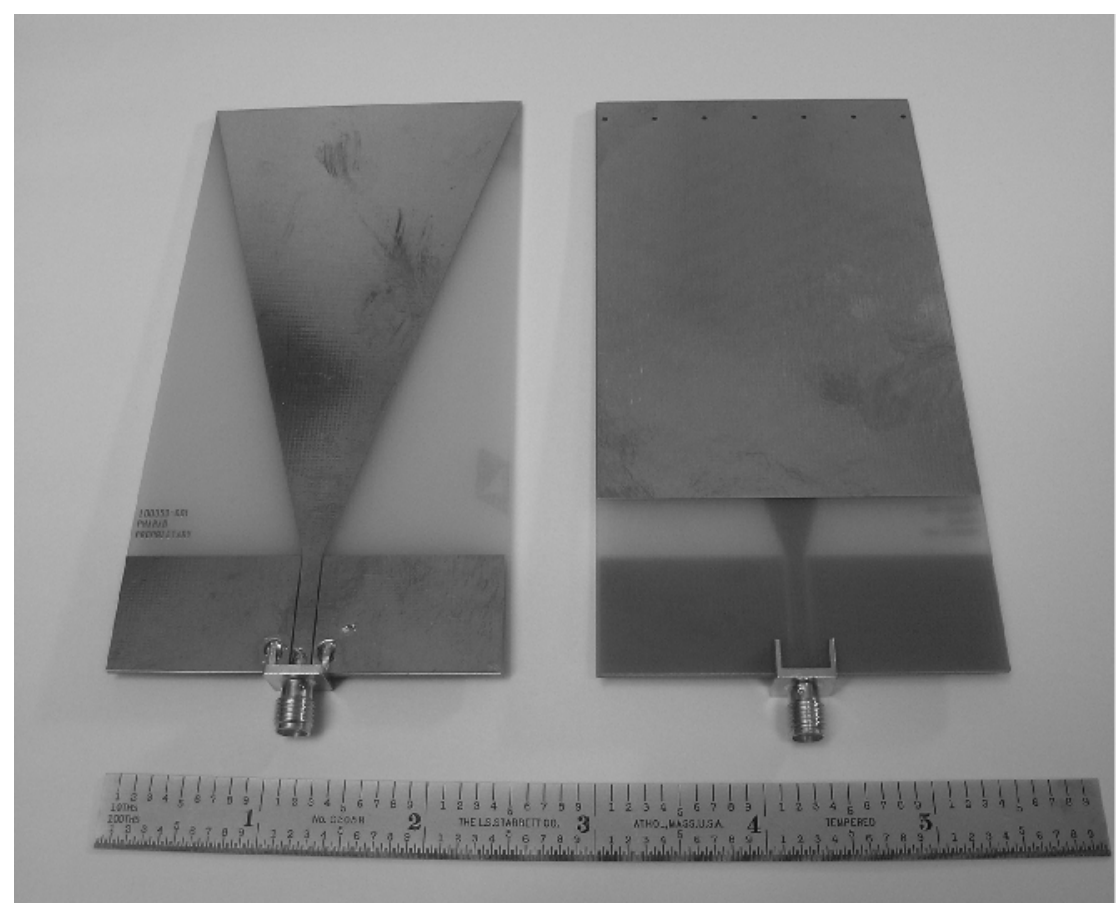

Figure 1.26 Photograph of a three-dimensional flared monopole [36].

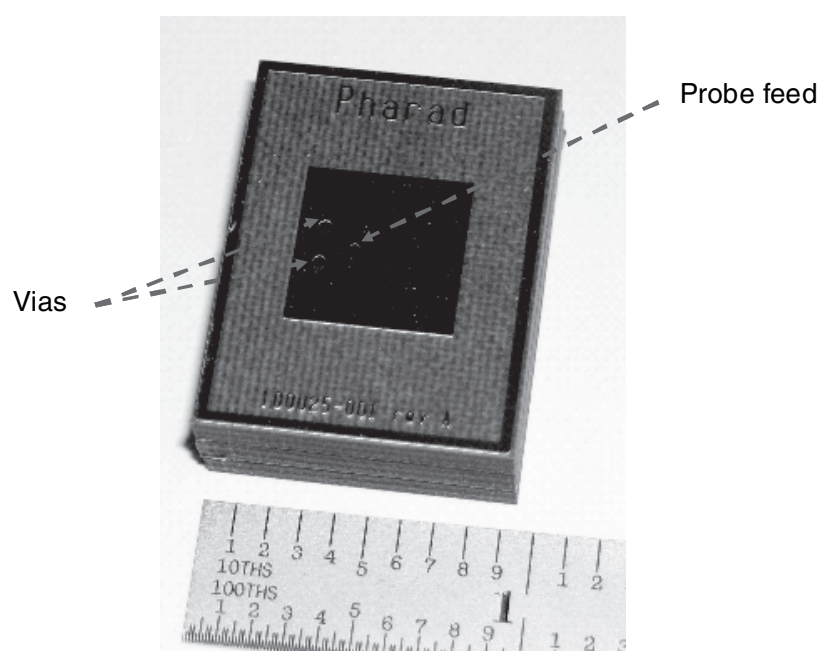

Figure 1.27 Photograph of a shorted patch antenna.

the number of publications in this area of R\&D there will be many more to come. It is the authors' opinion that no one technique gives the ultimate performance of a small radiator and really it is the environment in which the radiator is to be mounted that plays a significant role in which is the best size-reduction method. 


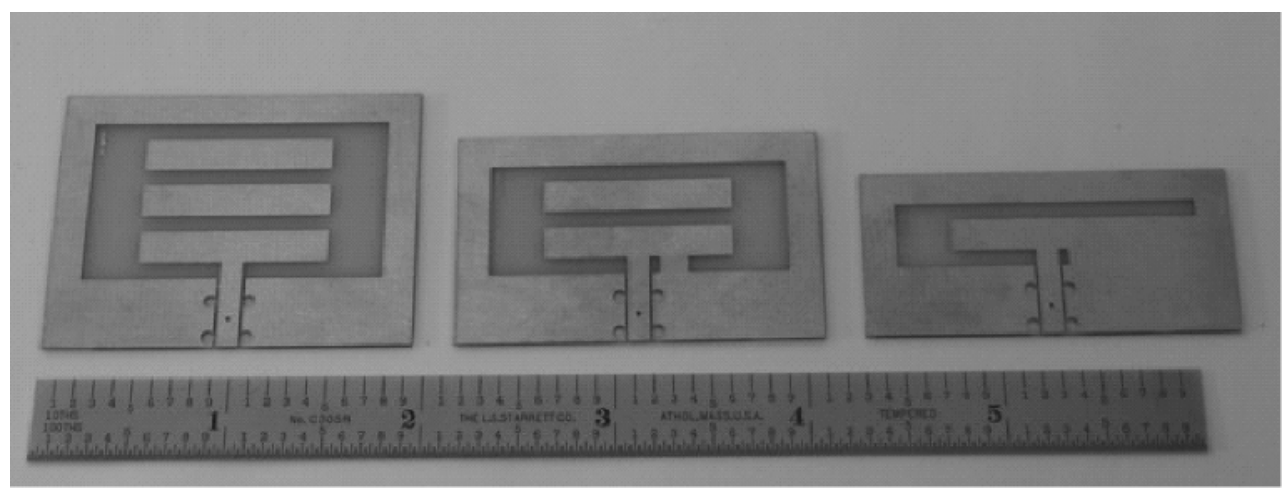

Figure 1.28 Photograph of printed folded slots [38].

\subsection{OUTLINE OF THE BOOK}

In this book several concepts related to wireless systems and printed antennas are presented. The remainder of the book is divided into three parts: Part I, Fundamental Wideband Printed Antennas for Wireless Systems; Part II, Small Printed Antennas for Wireless applications; and Part III, Advanced Concepts and Applications in Wireless Systems. The first part describes wideband printed antenna radiators suitable for a variety of wireless systems. In Chapter 2 multilayered patch antennas are presented including aperture coupled and proximity coupled patches. Stacked patches are investigated and design strategies and bandwidth optimization procedures are given. In Chapter 3 variations of printed quasi-Yagi antennas are discussed and several designs presented, including a case for operation at $120 \mathrm{GHz}$. Examples of arrays of these antennas are also given. These two chapters represent wideband variations of resonant-style printed antennas as previously discussed. In the next chapters travelling wave versions of printed antennas are explored. In Chapter 4 printed spiral antennas are presented and design strategies and a parameter study are given. In Chapter 5 a variation of the printed spiral, the printed folded Beverage antenna, is discussed and several examples given, including three-dimensional versions. In Chapter 6 the characteristics of tapered slot antennas are presented and, once again, there is a focus on looking at design trends so that the reader can establish how to optimize the performance of this class of radiator. Chapters 2 to 6 represent most of the wideband fundamental radiating elements that are presently being considered for wireless systems.

The next part of the book presents four chapters focusing on small printed antennas. In Chapter 7 several versions of PIFAs are examined, including multiband versions, and the impact of capacitive and inductive loading and fractal forms are summarized. In Chapter 8 a variety of shorted patches are presented including circular polarized and multiband versions. Techniques to reduce cross-polarization and also procedures to further reduce the conductor size of the antenna are summarized in this chapter. Chapter 9 looks at printed antenna specifically designed for WLAN applications, in particular versions of the printed monopole. Antennas capable of operating over several wireless communication system bandwidths are presented and a surface-mounted spiral printed monopole is examined. Chapter 10 presents how printed antennas can be incorporated into computers and other wireless communication components. In particular, this chapter shows how the performance of the antenna can be impacted by the surrounding environment. 
In the third part of the book some advanced concepts and applications in wireless systems are presented. Not only variations of printed antennas but also advanced EM and wireless concepts that can impact printed antenna designs are discussed. In Chapter 11 reflectarray antennas are presented, including the principles of operation and design methods of passive and active printed reflectarray antennas. This chapter also presents designs for automotive radar systems, base stations, and spatial power combiners. Chapter 12 looks at the use of artificial magnetic conductors and other surfaces and how they can be used to improve the performance of printed antennas. Chapter 13 presents an overview of integrated transceivers. Integrated transceivers are a very good example of where printed antennas are extremely useful. Chapter 14 investigates the art of reconfigurable antennas for a software defined radio. Here the radiators are designed to be inherently matched to the active elements feeding them. Once again this is an excellent example of where printed antennas can be very useful radiators, as the control of the input impedance of a printed antenna (especially a patch antenna) is easily controllable. Finally, Chapter 15 examines multiple-input multiple-output (MIMO) systems and how patch antennas can be used in these wireless systems.

\section{REFERENCES}

1. R. Prasad and L. Munoz, WLAN and WPANS towards 4G, Artech House, Norwood, Massachusetts, 2003.

2. D. M. Pozar, Microwave and RF Design of Wireless Systems, John Wiley \& Sons, Inc., New York, 2000.

3. E. D. Kaplan and C. Hegarty (eds), Understanding GPS: Principles and Applications, 2nd edn, Artech House, Norwood, Massachusetts, 2006.

4. J. D. Kraus and D. A. Fleisch, Electromagnetics with Applications, 5th edn, McGraw-Hill, New York, 1999.

5. Memorandum on Draft Policy for Radio Frequency Identification (RFID), DoD Acquisition, Technology and Logistics, 2 October 2003.

6. Memorandum on Update on Draft Policy for Radio Frequency Identification (RFID), DoD Acquisition, Technology and Logistics, 20 February 2004.

7. www.schreiner-online.com/en/html/presse/06_LogiData/06_3_rfid-Label/Transponder_und_ Etiketten.html

8. http://jtrs.army.mil/

9. Joint Tactical Radio System Operational Requirements Document (Extract of JROC approved final with waveform Table 4-2 and Annex E), Version 3.2, April 2003.

10. A. J. Goldsmith and S. B. Wicket, 'Design challenges for energy-constrained ad hoc wireless networks', IEEE Wireless Communications, 8-27, August 2002.

11. C. de Morais Cordeiro, H. Gossain and D. P. Agrawal, 'Multicast over wireless mobile ad hoc networks: present and future directions', IEEE Network, 52-59, January/February 2003.

12. S. Bellofiore, J. Foutz, R. Govindarajula, I. Bahceci, C. A. Balanis, A. S. Spanias, J. M. Capone and T. M. Duman, 'Smart antenna system analysis, integration and performance for mobile ad-hoc networks (MANETs)', IEEE Transactions on Antennas and Propogation, 50, 571-581, May 2002.

13. R. Ramanathan, 'Making ad hoc networks density adaptive', in Proceedings of MILCOM, 957-961, October 2001.

14. http://wwww.xtarllc.com/capabilities/indelx.htm

15. http://msl.jpl.nasa.gov/Programs/gps.html

16. http://grouper.iee.org/groups/802/11/

17. http://www.ieee802.org/15/pub/SG3c.html

18. http://grouper.ieee.org/groups/80215/

19. ITU Recommendation ITU-R P.676-3, 'Attenuation by atmospheric gases', Geneva, 1997. 
20. http://www.northropgrumman.com/index.html

21. http://www.aerosonde.com/

22. http://www.bluefinrobotics.com/

23. 'IEEE standard definitions of terms for antennas', IEEE Std 145-1983, IEEE Transactions on Antennas and Propagation, 31, November 1983.

24. K. S. Yngvesson et al., 'The tapered slot antenna - a new integrated element for millimeter-wave applications', IEEE Transactions on Microwave Theory and Techniques, 37, 365-374, February 1989 (invited paper).

25. K. S. Yngvesson et al., 'Endfire tapered slot antennas on dielectric substrates', IEEE Transactions on Antennas and Propagation, 33, 1392-1400, December 1985.

26. C. H. Chio and D. H. Schaubert, 'Parameter study and design of wide-band widescan dual-polarized tapered slot antenna arrays', IEEE Transactions on Antennas and Propagation, 48, 879-886, June 2000.

27. M. C. Greenberg, K. L. Virga and C. L. Hammond, 'Performance characteristics of the dual exponentially tapered slot antenna (DETSA) for wireless communications applications', IEEE Transactions on Vehicular Technology, 52, 305-312, March 2003.

28. R. B. Waterhouse, 'Traveling wave antennas', in Encyclopedia of RF and Microwave Engineering (ed. K. C. Chang), Vol. 6, John Wiley and Sons, Inc., New York, pp. 5363-5373, 2005.

29. J. Dyson, 'The equiangular spiral antenna', IRE Transactions on Antennas and Propagation, AP-7, 181-187, April 1959.

30. J. J. H. Wang and V. K. Tripp, 'Design of multioctave spiral-mode microstrip antennas', IEEE Transactions on Antennas and Propagation, 39, 332-335, March 1991.

31. S. D. Targonski, R. B. Waterhouse and D. M. Pozar, 'Design of wideband aperture-stacked patch microstrip antennas', IEEE Transactions on Antennas and Propagation, 46, 1246-1251, September 1998.

32. H.-S. Tsai and R. A. York, 'FDTD analysis of CPW-fed folded slot and multiple-slot antennas on thin substrates', IEEE Transactions on Antennas and Propagation, 44, 217-226, February 1996.

33. C. A. Balanis, Antenna Theory: Analysis and Design, 2nd edn, John Wiley \& Sons, Inc., New York, 1997.

34. R. B. Waterhouse, Microstrip Patch Antennas: A Designer's Guide, Kluwer, Boston, 2003.

35. L. C. Godara (ed.), Handbook on Antennas in Wireless Communications, CRC Press, New York, 2001.

36. R. B. Waterhouse and D. Novak, 'Printed folded flared monopole antenna', IEE Electronics Letters, 42, February 2006.

37. R. B. Waterhouse, S. D. Targonski and D. M. Kokotoff, 'Design and performance of small printed antennas', IEEE Transactions on Antennas and Propogation, 46, 1629-1633, November 1998.

38. R. Waterhouse and D. Novak, 'Small folded CPW fed slot antennas', in Proceedings of the IEEE Antennas and Propagation Symposium, Albuquerque, New Mexico, July 2006. 
